\title{
Realizing Joint Radar-Communications in Coherent MIMO Radars
}

\author{
Muharrem Arik ${ }^{\mathrm{a}}$, Ozgur B. Akan ${ }^{\mathrm{a}, \mathrm{b}}$ \\ ${ }^{a}$ Next-generation and Wireless Communications Laboratory (NWCL) \\ Department of Electrical and Electronics Engineering \\ Koc University, Istanbul, 34450, Turkey \\ ${ }^{b}$ Internet of Everything (IoE) Group \\ Electrical Engineering Division, Department of Engineering \\ University of Cambridge, Cambridge, CB3 OFA, UK
}

\begin{abstract}
The increasing interest on spectrum resources causes various efforts on developing smart and compact solutions as joint radarcommunication (JRC) systems. A JRC system can offer cost-effective solution with concurrent operation, as target sensing via radar processing and establishing communication links. JRC capability has been proposed over the years for different types of MIMO radars. However, a JRC capable monostatic coherent MIMO radar system is yet to be developed. These radars offer several advantages as fully coherent signal processing and coherent transmit beamforming which provides beampatterns to minimize probability of intercept. In this paper, two new waveform generation techniques suitable for JRC operation without disturbing transmit beamforming requirements and waveform orthogonality condition in space and time domain are proposed for monostatic coherent MIMO radars. Then, new communication methods are introduced for phase coded monostatic coherent MIMO radars. First method uses chirp-wise information encoding inside the radar pulse as intra-pulse communications. Second rotates the phase of a specific waveform on radiated symbols to a specific direction and the last method applies a small amount of progressive phase shift to the radar waveforms emitted from the antennas to create relative phase modulation between selected radar waveforms. Then, the performance of the proposed communication techniques are investigated in terms of bit error rate (BER) and generated waveforms are examined according to the orthogonality and transmit beamforming requirements.
\end{abstract}

Keywords: Joint Radar-Communication, Coherent MIMO Radar, Orthogonal Waveform Generation.

\section{Introduction}

Recently, a huge attention is attracted to the concept of integrating communication and radar missions within the same waveform. A JRC system can provide cost-effective platform solution with simultaneous operation, i.e. environment sensing via radar processing and establishment of communication links.

Several JRC techniques are proposed over the years for different types of radars with different topologies [1]. The oldest technique is embedding information into radar chirps which has been studied in [2,3]. In these methods, the information symbols can be transmitted to the direction of interest via emitting one waveform during each radar pulse from a group of predetermined waveforms. However, changing the emitted radar waveform from pulse to pulse may affect the primary radar operation in its coherent processing interval (CPI).

Various JRC methods for phased array radars which exploits the time modulated arrays (TMA) approach for amplitude modulation (AM), phase-shift-keying (PSK) and quadrature AM (QAM) based information embedding were proposed and named as Dual-Function Radar-Communication (DFRC).

Email addresses: marik@ku.edu.tr (Muharrem Arik), akan@ku.edu.tr, oba21@cam.ac.uk (Ozgur B. Akan)

URL: https ://nwcl.ku.edu.tr/marik.html (Muharrem Arik), https://ioe.eng.cam.ac.uk/directory/akan (Ozgur B. Akan)
Firstly proposed DFRC system is introduced for phased array radars with AM $[4,5]$. To provide variations in the sidelobe levels (SLLs) towards the communication direction, each phase of the transmit array of the phased array system is manipulated from pulse to pulse basis. For each pulse, the receiver only detects the amplitude of the received signal with comparing to a threshold and decipher the transmitted bit sequence. Phased array radar systems are considered to be single-input singleoutput (SISO) systems. On the other hand, for multiple-input multiple-output (MIMO) radars, transmit beamforming vectors each assigned to a orthogonal waveform can be utilized for reach more data rate. In this concept, radiated signal towards communication receiver from the MIMO radar aperture is modulated in a coherent or non-coherent manner. Information can be broadcasted or transmitted to a single or multiple direction.

In [6], a sidelobe amplitude shift keying (ASK) based MIMO DFRC system with dual-level amplitude control was introduced. Each bit is assigned to one orthogonal waveform. The information is modulated coherent manner, hence the receiver needs a channel estimation process to specify an effective threshold. Then, non-coherent sidelobe ASK based MIMO DFRC system is proposed in [7]. In this work, two orthogonal waveforms are dedicated to delivering one bits of information. Then, receiver decides corresponding bit via comparing the ratio of the matched filter outputs of the related orthogonal wave- 
forms. Moreover, a comprehensive overview of the information embedding techniques for the DFRC methods are given in [8]. One more sidelobe ASK based method proposed in [9]. In this work, a single dual orthogonal waveform is utilized for non data aided channel estimation process to form a threshold level under fading channel conditions. However, all of these sidelobe ASK based MIMO DFRC systems have some limitations that the communication receivers must be outside the radar mainlobe, which are not guaranteed in real world applications and their data rate is low.

Coherent [10, 11] and non-coherent [11, 12] PSK based MIMO DFRC methods for embedding information into the radar emission were recently proposed. During each radar pulse, the PSK based method embeds information using one phase symbol into the radar emission toward the communication angle. At the receiver, a phase detector is used to detect the embedded symbol using the outputs of the matched filters of the waveforms. Then, the receiver interprets the corresponding bit sequence. Unlike the sidelobe ASK based methods, PM-based DFRC methods are well suited for both directional communications and also broadcasting.

Recently, a sidelobe QAM based DFRC system is introduced in [13] for multiple antenna sub-arrays. Each sub-array is capable of transmitting an orthogonal waveform. In this work, if the communication receiver is in the radar sidelobe, information embedding is done with a novel QAM symbol mapping. However, if the receiver in mainlobe, then, non-coherent PSK based MIMO DFRC system is utilized. Using this QAM symbol constellation, although radar waveforms with medium pulse repetition frequency are used, high data rates seem achievable. Higher data rates are also achieved via another technique called far-field radiated emission design (FFRED) detailed in $[14,15]$. In this technique, the radar beam and the communication beam are in different directions. Hence, the transmitted power is smartly shared between radar and communication purposes, hence a loss in transmit power is occurred.

All of these techniques are investigated and evaluated so far under the main assumption that the transmitting radar waveforms are completely orthogonal to each other. None of them considers waveform design. Besides, perfectly orthogonal waveforms are hard to achieve. According to the recent research in [16], it is possible to find waveforms which satisfy almost orthogonality in time domain and transmit beamforming constraint in space domain simultaneously for a phase coded monostatic coherent MIMO radar structure. Monostatic coherent MIMO radars have several advantages over other types of MIMO radars. Hence, fully coherent signal processing can be feasible when element spacing comparable with wavelength. Moreover, coherent transmit beamforming offers beampatterns with nulls in certain directions to minimize probability of intercepting from reconnaissance receiver and lower the radiation from an interference or clutter.

In this work, first, MIMO radar waveform coding technique in [16] is modified for establishing more stable communication channel. Then, information embedding is realized for coherent MIMO radar without disturbing the orthogonality and transmit beamforming requirements. Three different communication
Table 1: List of major symbols and notations

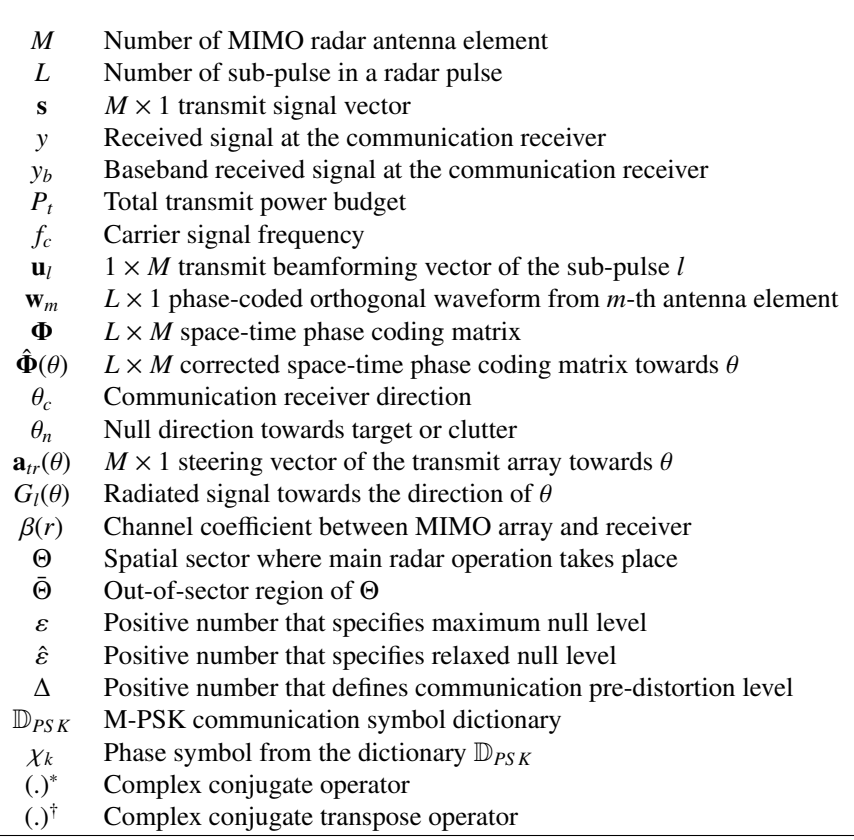

methods are proposed. In the first method, information symbols are embedded into radar waveforms without any decrease on orthogonality and they are transmitted only to a direction of interest. This method may provide thousands of bits per radar pulse. However, radar waveforms emitted from each MIMO antenna are revised by information, pulse by pulse. Hence, inter-pulse coherency may be limited using this method. Second method rotates the phase of a predetermined information vector which is only visible at communication direction. The phase of the information vector is modulated for each radar pulse for communication purposes and information bits are buried into transmit radiation pattern. Last method exploits the progressive phase difference between orthogonal waveforms emitted from the first and the last antenna element caused by a small degrees of steering on transmit beampattern. Last two methods aim to provide transparency over radar sensing operations and fully inter-pulse coherent processing can be applied. For this purpose, all radar waveforms emitted from antenna elements are preserved over each coherent processing interval (CPI) and the antenna locations of the emitting waveforms are remained the same. However, data rate is limited to a single symbol per pulse. For each radar pulse, both methods embed only one communication symbol during the operation and only the second method is requires coherency between JRC enabled transmitter platform and the receiver.

The remainder is organized as follows. Firstly, the signal model is given in Sec. II. Then, the space-time waveform coding and orthogonality for communication is detailed in Sec. III and the proposed information embedding methods are introduced in Sec. IV. Sec. V provides the performance evaluation results. Lastly, conclusions are discussed in Sec. VI. For ease of exposition, the major notations used throughout this paper are listed in Table 1. 


\section{Signal Model}

JRC system is equipped with $M$ MIMO radar antennas aligned as a uniform linear array (ULA). In the array, each element has an isotropic radiation pattern with spacing distance of $d$ in terms of a wavelength as shown in Fig. 1. Also, each of the antenna element in a MIMO array has a capability transmitting distinct phase coded quasi-orthogonal waveforms at the same carrier frequency. Although, there are several techniques for generating orthogonal MIMO radar waveforms, phase coding is the simplest one to implement. Therefore, phase coding method is considered for designing orthogonal waveforms in this work.

$M \times 1$ transmit signal vector during the $l$-th sub-pulse of the $r$-th radar pulse is,

$$
\mathbf{s}(t ; r ; l)=\mathbf{u}_{l}^{\dagger}(r) s_{c}(t), l=1,2, \ldots, L,
$$

where $r$ and $l$ are the radar pulse and sub-pulse index, respectively and $t$ is the fast time index, (. $)^{\dagger}$ stands for the complex conjugate transpose and carrier signal can be given as $s_{c}(t)=\sqrt{P_{t} / M} e^{j 2 \pi f_{c} t}$ where $P_{t}$ is the total transmit power budget and $f_{c}$ is the radar carrier signal. Then, $\mathbf{u}_{l}$ is the $1 \times M$ transmit beamforming weight vector of the sub-pulse $l$ as,

$$
\mathbf{u}_{l}=\left[e^{j \varphi_{1}(l)}, e^{j \varphi_{2}(l)}, \ldots, e^{j \varphi_{M}(l)}\right], l=1,2, \ldots, L,
$$

where $\varphi_{m}(l)$ is the phase of the sub-pulse $l$ from the antenna element $m$. For the simplicity, we can ignore the carrier frequency component, hence it does not have any affect on the final result. Then, transmitted phase coded orthogonal waveform from $m$-th antenna element for each radar pulse is given as,

$$
\begin{gathered}
w_{m}(t)=\sum_{l=1}^{L} \operatorname{Rect}\left[\frac{t-(l-0.5) \tau}{\tau}\right] w_{m}^{l}, \\
m=1,2, \ldots, M
\end{gathered}
$$

where $\tau$ is the coded waveform sub-pulse duration, $w_{m}^{l}$ be the $l$-th sub-pulse of the coded waveform for all radar pulses in a coherent processing interval from the antenna element $m$, and Rect(.) is the unit rectangular function as,

$$
\operatorname{Rect}(t)=\left\{\begin{array}{ll}
1, & 0 \leq t \leq \tau \\
0, & \text { otherwise }
\end{array} .\right.
$$

The $M \times 1$ transmitted phase coded orthogonal waveform vector $\mathbf{w}(t)$ can be written as,

$$
\mathbf{w}(t)=\left[w_{1}(t), w_{2}(t), \ldots, w_{M}(t)\right]^{T},
$$

from (2), orthogonal phase coded waveform can also be represented as a vector,

$$
\mathbf{w}_{m}=\left[e^{j \varphi_{m}(1)}, e^{j \varphi_{m}(2)}, \ldots, e^{j \varphi_{m}(L)}\right]^{T}, m=1,2, \ldots, M
$$

For each radar pulse, $L \times M$ space-time phase coding matrix for $M$ antennas and $L$ sub-pulses can be given as,

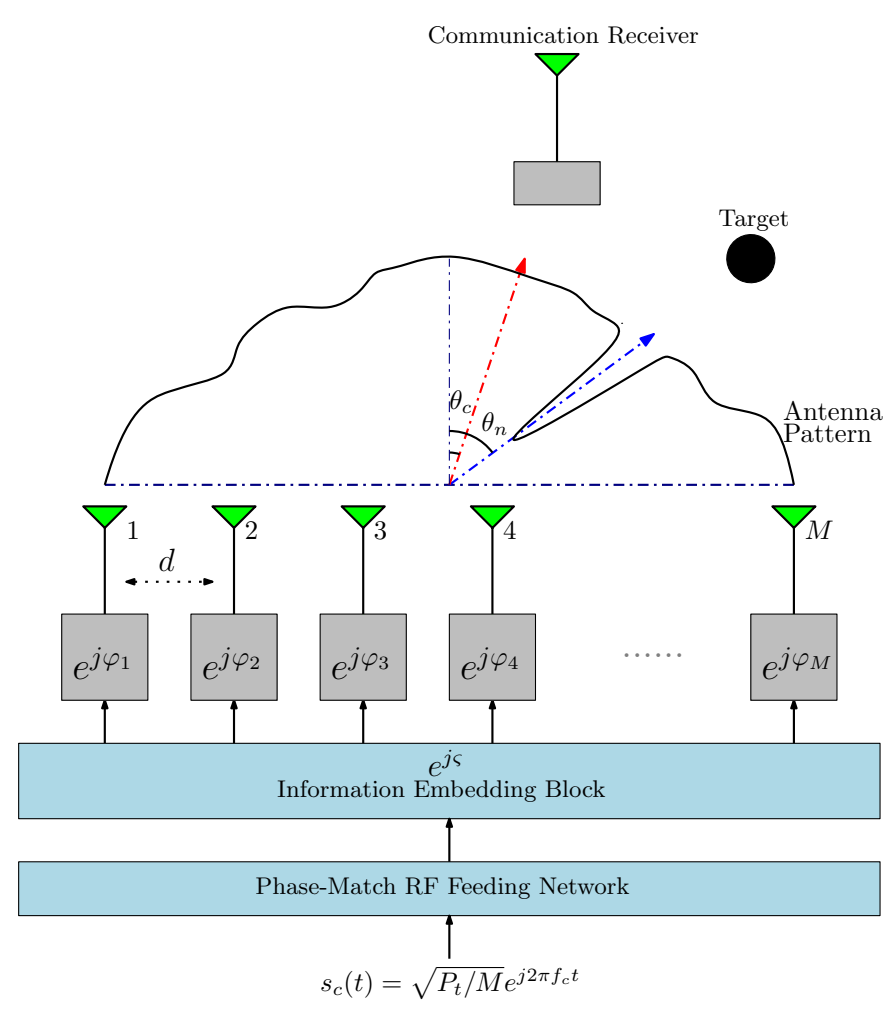

Fig. 1: JRC enabled Coherent MIMO radar transmitter architecture.

$$
\begin{aligned}
\boldsymbol{\Phi} & =\angle \boldsymbol{\Phi}=\left[\begin{array}{c}
\angle \mathbf{u}_{1} \\
\angle \mathbf{u}_{2} \\
\vdots \\
\angle \mathbf{u}_{L}
\end{array}\right]=\left[\begin{array}{c}
\angle \mathbf{w}_{1} \\
\angle \mathbf{w}_{2} \\
\vdots \\
\angle \mathbf{w}_{M}
\end{array}\right]^{T} \\
& =\left[\begin{array}{cccc}
\varphi_{1}(1) & \varphi_{2}(1) & \ldots & \varphi_{M}(1) \\
\varphi_{1}(2) & \varphi_{2}(2) & \ldots & \varphi_{M}(2) \\
\vdots & \vdots & \vdots & \vdots \\
\varphi_{1}(L) & \varphi_{2}(L) & \ldots & \varphi_{M}(L)
\end{array}\right],
\end{aligned}
$$

where $L$ is the phasor angle function. It should be noted that, there is a relation between $\mathbf{w}_{m}$ and $\mathbf{u}_{l}$, as $w_{m}^{l}=u_{l}^{m}$. Then, each phase coded waveform waveform is normalized to have unit power, i.e. $\int_{T_{P W}}\left|s_{m}(t ; r ; l)\right|^{2} d t=1$ for $m=1, \ldots, M$, where $T_{P W}=$ $\tau L$ is the radar pulse width.

At the receiver side, a single omni-directional antenna element is connected to a communication receiver located in direction $\theta_{c}$, which is assumed to be known from the receiver. Besides, JRC enabled communication receiver must be equipped with a matched filter. The received signal at the communication receiver for sub-pulse $l$ can be expressed as,

$$
y(t ; r ; l)=\beta(r) \mathbf{a}_{t r}^{T}\left(\theta_{c}\right) \mathbf{s}(t ; r ; l)+\eta(t ; r ; l),
$$

where $\mathbf{a}_{t r}\left(\theta_{c}\right)$ is the $M \times 1$ steering vector of the transmit array toward the spatial angle $\theta_{c}, \beta(r)$ is the channel coefficient which reflects the propagation gain between the transmit coherent MIMO array and the communication receiver during the $r$ th 
pulse. It is assumed that the channel is same during the radar pulse. Then, $\eta(t ; r ; l)$ is the additive white Gaussian noise with zero mean and variance $\sigma_{N}^{2}$. The baseband received signal for sub-pulse $l$ can be written as,

$$
y_{b}(t ; r ; l)=\beta(r) \sqrt{P_{t} / M} \mathbf{a}_{t r}^{T}\left(\theta_{c}\right) \mathbf{u}_{l}^{\dagger}(r)+\hat{\eta}(t ; r ; l),
$$

and,

$$
\mathbf{a}_{t r}(\theta)=\left[1, e^{-j 2 \pi d \sin (\theta)}, \ldots, e^{-j 2 \pi(M-1) d \sin (\theta)}\right]^{T}
$$

where $\hat{\eta}(r)$ is the noise at the output of the matched filter whose variance is the same as that of $\eta(t ; r)$ and $(.)^{T}$ stands for the transpose operations. $P_{t}$ is selected as $M$ for the rest of the paper. Then, the baseband signal for radar pulse $r$ can be expressed as,

$$
y_{b}(t ; r)=\beta(r) \sum_{l=0}^{L-1} \operatorname{Rect}\left[\frac{t-(l+0.5) \tau}{\tau}\right] G_{l}\left(\theta_{c}\right)+\eta(t ; r),
$$

where $G_{l}\left(\theta_{c}\right)=\mathbf{a}_{t r}^{T}\left(\theta_{c}\right) \mathbf{u}_{l}^{\dagger}(r)=\mathbf{u}_{l}^{*}(r) \mathbf{a}_{t r}\left(\theta_{c}\right)$ and $(.)^{*}$ is the conjugate operation. Using information embedding strategies via manipulating transmit signal vector $\mathbf{s}(t ; r)$, communication symbols can be buried into the radar signal.

\section{Space-Time Waveform Coding and Orthogonality For Communication}

While satisfying the transmit beamforming requirements in space domain, we must ensure waveform orthogonality between MIMO antenna elements in time domain. More to these, for robust communication purposes more requirement must be added. Then, two different methods are proposed based on the technique in [16]. In each method, each row of (7) is derived iteratively using the phases of the first sub-pulse transmit weight vector $\mathbf{u}_{1}$. Please note that, these methods must be operated in offline.

In order to comply communication requirements, first, transmit beamforming vectors, $\mathbf{u}$, must be smartly selected to satisfy to focus the transmit power within the radar main beam or deepest nulls in certain directions, while keeping the sidelobe levels deviates under a certain value.

\subsection{Transmit Beamforming Design}

We have considered the most practical transmit beamforming requirement as one null in an arbitrary direction $\theta_{n}$ in which a target, clutter or a another radar could be located. Off-line methods in the literature generally uses genetic algorithms (GA) $[6,7]$. We have also used GA algorithms to form first sub-pulse transmit weight vector $\mathbf{u}_{1}$ in off-line solving the following optimization problem using phase-only synthesis,

$$
\begin{gathered}
\min _{u_{k}} \max _{\theta}|1-| \mathbf{u}_{k}^{*} \mathbf{a}_{t r}(\theta)||, \theta \in \bar{\Theta}, \\
\text { s.t. }\left\|\mathbf{u}_{k}^{*} \mathbf{a}_{t r}\left(\theta_{n}\right)\right\| \leq \varepsilon, \theta_{n} \in \Theta,
\end{gathered}
$$

where $\mathbf{u}_{k}$ be the $1 \times M$ transmit beamforming weight vectors for first sub-pulse, $\Theta$ is the spatial sector for the desired null and $\theta_{n}$

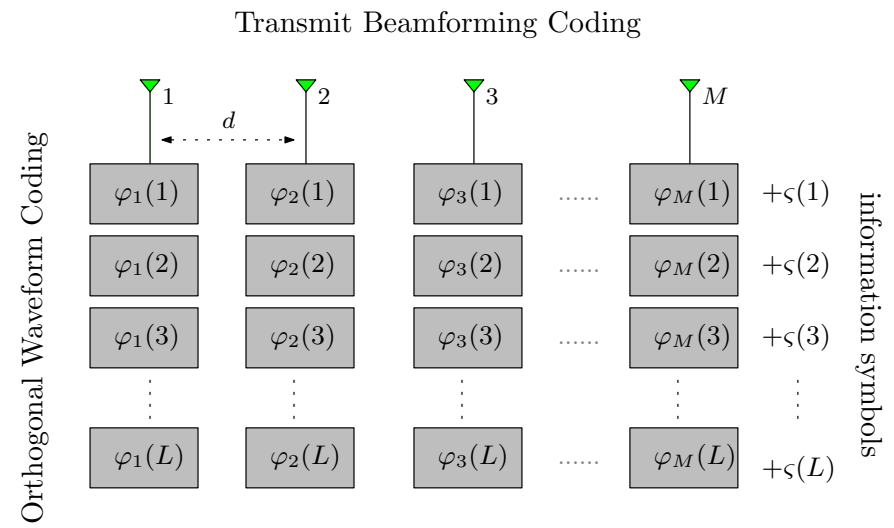

Fig. 2: Space-Time Code Matrix

is the desired null direction. $\bar{\Theta}$ represents out-of-sector region of the null sector $\Theta$ and $\varepsilon$ is a positive number that specifies maximum null level.

In order to avoid nulls at communication direction $\theta_{c}$ outside the null sector, several beam-patterns may be synthesized for effective JRC operation using the GA based off-line method. Then, according to the required communication direction, the first sub-pulse transmit weight vector $\mathbf{u}_{1}$ is selected as $\max _{\mathbf{u}_{k}}\left\|\mathbf{u}_{k}^{*} \mathbf{a}_{t r}\left(\theta_{c}\right)\right\|$. Communication direction is assumed to be constant in each coherent processing interval, and can change in the next CPI.

\subsection{Null Direction Fixed Waveform Coding}

First method aims to fix the radiation signal at null direction using the technique in $[16,17]$. This technique promises no guarantee that secures higher signal levels for the communication direction. The technique in $[16,17]$ fixes the null location at $\theta_{n}$ by modifying $\mathbf{u}_{l}$ in (2) as,

$$
\mathbf{u}_{l+1}=\left[\begin{array}{c}
e^{\left[\varphi_{I_{1}}(l)+2 \pi\left(1-I_{1}\right) d \sin \theta_{n}\right]} \\
e^{\left[\varphi_{I_{2}}(l)+2 \pi\left(2-I_{2}\right) d \sin \theta_{n}\right]} \\
\vdots \\
e^{\left[\varphi_{\left.I_{M}(l)+2 \pi\left(M-I_{M}\right) d \sin \theta_{n}\right]}\right.}
\end{array}\right]^{T} \text { for } l=2,3, \ldots, L
$$

where $\mathbf{u}_{l+1}$ be the modified version of transmit beamforming weight vectors for the $l$ th sub-pulse, $\left\{I_{1}, I_{2}, \ldots, I_{M}\right\}$ are the indexes of a random permutation sequence of $\{1,2, \ldots, M\}$ and $\varphi_{I_{m}}(l)$ is the new phase component of the $m$-th antenna element for the $l$-th sub-pulse. The radiated signals of $\mathbf{u}_{l}$ and $\mathbf{u}_{l+1}$ at $\theta_{n}$ can be given as,

$$
\begin{aligned}
G_{l}\left(\theta_{n}\right)= & {\left[e^{j \varphi_{1}(l)}, e^{j \varphi_{2}(l)}, \ldots, e^{j \varphi_{M}(l)}\right] } \\
& \times\left[1, e^{-j 2 \pi d \sin \left(\theta_{n}\right)}, \ldots, e^{-j 2 \pi(M-1) d \sin \left(\theta_{n}\right)}\right]^{T} \\
= & \sum_{m=1}^{M} e^{j\left[\varphi_{m}-2 \pi(m-1) d \sin \theta_{n}\right]}=\sum_{m=1}^{M} e^{j\left[\varphi_{m}-\varrho(m-1)\right]},
\end{aligned}
$$




$$
\begin{aligned}
G_{l+1}\left(\theta_{n}\right)= & {\left[\begin{array}{c}
e^{j\left[\varphi_{I_{1}}(l)+2 \pi\left(1-I_{1}\right) d \sin \theta_{n}\right]} \\
\vdots \\
e^{j\left[\varphi_{I_{M}}(l)+2 \pi\left(M-I_{M}\right) d \sin \theta_{n}\right]}
\end{array}\right]^{T} } \\
& \times\left[1, e^{-j 2 \pi d \sin \left(\theta_{n}\right)}, \ldots, e^{-j 2 \pi(M-1) d \sin \left(\theta_{n}\right)}\right]^{T} \\
= & \sum_{m=1}^{M} e^{j\left[\varphi_{I_{m}}-2 \pi\left(I_{m}-1\right) d \sin \left(\theta_{n}\right)\right]}=\sum_{m=1}^{M} e^{j\left[\varphi_{I_{m}}-\varrho\left(I_{m}-1\right)\right]},
\end{aligned}
$$

where $\varrho=2 \pi d \sin \theta_{n}$. Hence, the expression $\sum_{m=1}^{M} e^{j\left[\varphi_{m}-\varrho(m-1)\right]}$ is equal to the $\sum_{m=1}^{M} e^{j\left[\varphi_{I_{m}}-\varrho\left(I_{m}-1\right)\right]}$, it is clear that the radiation at $\theta_{n}$ is the same for $\mathbf{u}_{l}$ and $\mathbf{u}_{l+1}$.

After each permutation on phases of all antenna elements, in order to avoid nulls or small signal levels at communication direction, the radiated signal $\left|G_{l+1}\left(\theta_{c}\right)\right|$ is examined that it is in the $\pm \Delta \mathrm{dB}$ neighborhood of $\left|G_{1}\left(\theta_{c}\right)\right|$. This constraint is given as,

$$
\frac{\left|G_{1}\left(\theta_{c}\right)\right|}{\Delta} \leq\left|G_{l+1}\left(\theta_{c}\right)\right| \leq \Delta\left|G_{1}\left(\theta_{c}\right)\right|,
$$

where $l=2, \ldots, L-1, \Delta$ is a positive number which is defined as communication pre-distortion level. Note that, $\Delta$ is equal to $10 \log _{10} 4$. (17) can be rewritten as,

$$
1 / \Delta \leq \frac{\left|\mathbf{u}_{l+1}^{*} \mathbf{a}_{t r}\left(\theta_{c}\right)\right|}{\left|\mathbf{u}_{1}^{*} \mathbf{a}_{t r}\left(\theta_{c}\right)\right|} \leq \Delta .
$$

If (18) not satisfied, permutation is repeated. The search is continued as updating $l<=l+1$, until to reach the last sub-pulse, when (18) is met. Then, $L \times M$ space-time phase coding matrix for $M$ antennas and $L$ sub-pulses in (7) is formed. It should be noted that, setting a smaller pre-distortion level causes extended search times for successive transmit beamforming patterns, although it improves the communication performance.

In order to emit communication symbol with fixed phase to the communication direction via beamforming, also the phase response must be corrected before information embedding. For the correction, (7) can be re-written as,

$$
\begin{aligned}
\hat{\mathbf{\Phi}}\left(\theta_{c}\right) & =\operatorname{diag}\left\{\boldsymbol{\varsigma}\left(\theta_{c}\right)\right\} \boldsymbol{\Phi} \\
& =\left[\begin{array}{cccc}
1 & 0 & \ldots & 0 \\
0 & e^{j \varsigma(2)} & \ldots & 0 \\
\vdots & \vdots & \vdots & \vdots \\
0 & 0 & \ldots & e^{j \zeta(L)}
\end{array}\right]\left[\begin{array}{c}
\mathbf{u}_{1} \\
\mathbf{u}_{2} \\
\vdots \\
\mathbf{u}_{L}
\end{array}\right] \\
& =\left[\begin{array}{c}
\mathbf{u}_{1} \\
e^{j((2)} \mathbf{u}_{2} \\
\vdots \\
e^{j \zeta(L)} \mathbf{u}_{L}
\end{array}\right]=\left[\begin{array}{c}
\boldsymbol{\varsigma} \odot \mathbf{w}_{1} \\
\boldsymbol{\varsigma} \odot \mathbf{w}_{2} \\
\vdots \\
\boldsymbol{\varsigma} \odot \mathbf{w}_{M}
\end{array}\right]^{T},
\end{aligned}
$$

where $\odot$ is the Hadamard element-wise product, $\boldsymbol{S}$ is a correction vector and $\operatorname{diag}\{\boldsymbol{\varsigma}\}$ is the $L \times L$ diagonal matrix whose entities are the elements of the vector $\boldsymbol{s}$. Also the correction vector $\boldsymbol{s}$ can be given as,

$$
\boldsymbol{S}\left(\theta_{c}\right)=\left[1, e^{j \zeta(2)}, \ldots, e^{j \zeta(L)}\right]^{T}, \varsigma(l)=\angle G_{1}\left(\theta_{c}\right)-\angle G_{l}\left(\theta_{c}\right)
$$

for $l=2, \ldots, L$.

\subsection{Communication Direction Fixed Waveform Coding}

Apart from the first method, second method aims to fix the radiation signal at communication direction via modifying the $\theta_{n}$ to $\theta_{c}$ in (14) as,

$$
\mathbf{u}_{l+1}=\left[\begin{array}{c}
e^{\left[\varphi_{I_{1}}(l)+2 \pi\left(1-I_{1}\right) d \sin \theta_{c}\right]} \\
e^{\left[\varphi_{I_{2}}(l)+2 \pi\left(1-I_{2}\right) d \sin \theta_{c}\right]} \\
\vdots \\
e^{\left[\varphi_{I_{M}}(l)+2 \pi\left(1-I_{M}\right) d \sin \theta_{c}\right]}
\end{array}\right]^{T},
$$

then, after each permutation on phases at antenna elements, resulting beam pattern is examined until the condition below,

$$
\left\|\mathbf{u}_{l+1}^{*} \mathbf{a}_{t r}\left(\theta_{n}\right)\right\| \leq \hat{\varepsilon}, \theta_{n} \in \Theta,
$$

where $\hat{\varepsilon}$ is a positive number and named as relaxed null level constant. If (22) not satisfied, permutation is repeated. This process is continued as updating $l<=l+1$ to reach last, $L$-th sub-pulse. Then, $L \times M$ space-time phase coding matrix for $M$ antennas and $L$ sub-pulses in (7) is formed.

However, if $\hat{\varepsilon}$ close to $\varepsilon$ in (13), it becomes harder to find $L$ number of beamforming vector which has deep null at $\theta_{n}$ as vector $\mathbf{u}_{1}$. Therefore, we must loose the null requirement to find more beamforming vector as $\hat{\varepsilon}=\varepsilon \delta$. $\delta$ is a positive number that specify the difference from the desired null.

\section{Proposed Information Embedding Methods}

In this section, three different information embedding methods for JRC enabled coherent MIMO radars are detailed. In the first communication technique, information symbols are embedded into all orthogonal waveforms as intra-pulse communications. Thus, radar waveforms are modified by information vector for each radar pulse, hence inter-pulse coherency will be limited. Last two methods aim to provide transparency over radar sensing operations. For this purpose, all radar waveforms are preserved over each CPI and emitting antenna locations are remained same. For each radar pulse, both methods embed only one communication symbol during the operation. Information bits are buried into radiation pattern for the second method and buried into dual waveform via M-ary phase-shift keying (MPSK) technique.

\subsection{Intra-Pulse Directional Communication}

The first method exploits the phase stability of the radiation towards the communication direction $\theta_{c}$ which is detailed in Sec. 3.2 and Sec. 3.3. These techniques offer a stable communication channel for a specific direction. Then, using this channel each sub-pulse can be modulated with differential MPSK technique as long as it does not violate the space-time orthogonality condition between MIMO radar waveforms.

Let $K$ bits of information is embedded for each radar subpulse $l$ via rotating the phase of the transmit beamforming vector $\mathbf{u}_{l}$ in (2), using the phase symbol $\chi_{k}$ from the M-PSK communication dictionary $\mathbb{D}_{P S K}=\left\{0, \frac{2 \pi}{K}, \ldots, \frac{(K-1) 2 \pi}{K}\right\}$. Then, the modulated transmit beamforming vector $\hat{\mathbf{u}}$ can be given as, 


$$
\hat{\mathbf{u}}_{r, l}=\left\{\begin{array}{ll}
\mathbf{u}_{l} & \text { for } l=1 \\
e^{j \gamma_{k}(r ; 1)} \mathbf{u}_{l} & \text { for } l=2 \\
e^{j\left(\gamma_{k}(r ; j-1)+\gamma_{k}(r ; j)\right)} \mathbf{u}_{l} & \text { for } l=3, \ldots, L
\end{array},\right.
$$

where $j=1, \ldots, L-1$ and $\gamma_{k}(r ; j)$ is the phase symbol for the $l$-th sub-pulse of the $r$-th radar pulse. After the radiated signal reached to the communication receiver omni-directional antenna, the baseband received signal (9) for $l$-th sub-pulse becomes,

$$
\begin{array}{r}
y_{b}(t ; r ; 1)=\beta(r) \hat{\mathbf{u}}_{r, l}^{*} \mathbf{a}_{t r}\left(\theta_{c}\right)+\eta(t ; r ; 1) \\
=\beta(r) \mathbf{u}_{l}^{*} \mathbf{a}_{t r}\left(\theta_{c}\right)+\eta(t ; r ; 1) \\
=\beta(r) G_{1}\left(\theta_{c}\right)+\eta(t ; r ; 1) \\
y_{b}(t ; r ; 2)=\beta(r) e^{j \gamma_{k}(r ; 1)} G_{2}\left(\theta_{c}\right)+\eta(t ; r ; 2) \\
y_{b}(t ; r ; l)=\beta(r) e^{j\left(\gamma_{k}(r ; j-1)+\gamma_{k}(r ; j)\right)} G_{l}\left(\theta_{c}\right)+\eta(t ; r ; l) \\
\text { for } l=3, \ldots, L,
\end{array}
$$

and $G_{l}\left(\theta_{c}\right)$ is equal to $G_{1}\left(\theta_{c}\right)$ for the null direction fixed waveform coding method detailed in Sec. 3.2. The receiver estimates the phase symbol as,

$$
\hat{\chi}_{k}(r, j)=\left\{\begin{array}{ll}
\operatorname{angle}\left(\frac{y_{b}(t ; r ; 2)}{y_{b}(t ; r ; 1)}\right) & \text { for } j=1 \\
\operatorname{angle}\left(\frac{y_{b}(t ; r ; l)}{y_{b}(t ; r ; l-1)}\right) & \text { for } j=2, \ldots, L-1
\end{array},\right.
$$

where $l=3, \ldots, L$. Then, the embedded binary sequence can be deciphered by comparing $y_{b}(t ; r ; l)$ signals which is obtained from (27) to the phase dictionary $\mathbb{D}_{P S K}$. Note that, this communication technique does not require coherency between the transmit MIMO array and the receiver. It is only assumed that the channel constant $\beta(r)$ is constant during the two consecutive sub-pulse.

\subsection{Code-Driven Secure Communication}

The second method is using coherent MIMO radiation pattern for communication and encodes it via an information vector during each radar pulse. Then, the phase of the encoded vector is rotated pulse by pulse for communication purposes.

After ensuring the orthogonality and communication stability at communication direction via space-time waveform coding techniques in Sec. 3.2 and Sec. 3.3, a pseudo-random coded communication vector $\gamma$ embedded into all orthogonal waveforms and then this communication waveform is phase modulated and becomes $\gamma_{k}$ via phase rotation of all waveforms $w_{m}$. The space-time phase coding matrix is modified with pseudorandom coded communication vector as,

$$
\hat{\boldsymbol{\Phi}}_{r}\left(\theta_{c}\right)=\operatorname{diag}\left\{\boldsymbol{\gamma}_{k}(r)\right\} \hat{\boldsymbol{\Phi}}\left(\theta_{c}\right),
$$

where $\gamma_{k}$ is the $L \times 1$ information encoded communication symbol vector and can be given by,

$$
\begin{aligned}
\gamma_{k}(r) & =e^{j \chi_{k}(r)} \gamma \\
& =e^{j \chi_{k}(r)}[\gamma(1), \gamma(2), \ldots, \gamma(L)]^{T},
\end{aligned}
$$

where $\chi_{k}$ is the phase symbol from the M-PSK communication dictionary $\mathbb{D}_{P S K}$ when $\log _{2} K$ bits of information is embedded for each pulse. Hence, $\gamma_{k}$ is the phase rotated version of communication vector $\gamma$. If the modified space-time coding matrix $\hat{\mathbf{\Phi}}_{r}\left(\theta_{c}\right)$ is used for radar operation, radiated signal towards communication direction can also be represented as,

$$
\begin{aligned}
\hat{\mathbf{s}}_{r} & =\hat{\mathbf{\Phi}}_{r}\left(\theta_{c}\right) \mathbf{a}_{t r}\left(\theta_{c}\right) \\
& =\operatorname{diag}\left\{\boldsymbol{\gamma}_{k}(r)\right\} \hat{\mathbf{\Phi}}\left(\theta_{c}\right) \mathbf{a}_{t r}\left(\theta_{c}\right) \\
& =\operatorname{diag}\left\{\boldsymbol{\gamma}_{k}(r)\right\}\left[\hat{G}_{1}\left(\theta_{c}\right), \hat{G}_{2}\left(\theta_{c}\right), \ldots, \hat{G}_{L}\left(\theta_{c}\right)\right]^{T} \\
& =e^{j \chi_{k}(r)}\left[\hat{G}_{1}\left(\theta_{c}\right) \gamma(1), \hat{G}_{2}\left(\theta_{c}\right) \gamma(2), \ldots, \hat{G}_{L}\left(\theta_{c}\right) \gamma(L)\right]^{T}
\end{aligned}
$$

where $\hat{G}_{l}\left(\theta_{c}\right)$ is equal to $G_{1}\left(\theta_{c}\right)$ for communication direction fixed technique and $\left|G_{l}\left(\theta_{c}\right)\right| / G_{1}\left(\theta_{c}\right)$ for null direction fixed technique. Then, $\hat{\mathbf{s}}_{r}$ can be rewritten as,

$$
\hat{\mathbf{s}}_{r}=e^{j \chi_{k}(r)} \gamma G_{1}\left(\theta_{c}\right)
$$

and if the technique in Sec. 3.2 is applied for space-time waveform coding, a distorted version of the communication vector $\gamma$ is used and it can be given as,

$$
\gamma=\left[\gamma(1), \frac{\left|G_{2}\left(\theta_{c}\right)\right|}{G_{1}\left(\theta_{c}\right)} \gamma(2), \ldots, \frac{\left|G_{L}\left(\theta_{c}\right)\right|}{G_{1}\left(\theta_{c}\right)} \gamma(L)\right]^{T} .
$$

After the radiated signal reached to the communication receiver, the baseband received signal for $l$-th sub-pulse becomes,

$$
y_{b}(t ; r ; l)=\beta(r) e^{j \chi_{k}(r)} \gamma(l) \mathbf{u}_{r, l}^{*} \mathbf{a}_{t r}\left(\theta_{c}\right)+\eta(t ; r ; l),
$$

then, for the $r$-th radar pulse,

$$
\begin{aligned}
y_{b}(t ; r)= & \beta(r) e^{j \chi_{k}(r)} G_{1}\left(\theta_{c}\right) \sum_{l=0}^{L-1} \operatorname{Rect}\left[\frac{t-(l+0.5) \tau}{\tau}\right] \gamma(l+1) \\
& +\eta(t ; r) \\
= & \beta(r) e^{j \chi_{k}(r)} G_{1}\left(\theta_{c}\right) \alpha(t)+\eta(t ; r),
\end{aligned}
$$

where $\alpha(t)=\sum_{l=0}^{L-1} \operatorname{Rect}\left[\frac{t-(l+0.5) \tau}{\tau}\right] \gamma(l+1)$ is the coded communication waveform during radar pulse. The baseband received signal is passed through the matched filter of $\alpha(t)$ and the output is written as,

$$
\hat{y}_{b}(r)=\beta(r) e^{j \chi_{k}(r)} G_{1}\left(\theta_{c}\right)+\hat{\eta}(r),
$$

where $\hat{\eta}(r)$ is the noise at the output of the matched filter whose variance is the same as that of $\eta(t ; r)$.

In practice, pilot and training sequences can be periodically transmitted to revise the estimated channel response. Then, using this training operation the system can provide phase synchronization between the coherent MIMO array and the communication receiver. Thus, let us assume that the channel is estimated correctly. In order to detect the embedded communication symbol $\chi_{k}$, the receiver estimates the phase in (35) with the perfect knowledge about the channel response $\beta$ and with 
the prior knowledge of $G_{1}\left(\theta_{c}\right)$. Then, $\chi_{k}(r)$ is estimated via calculating the angle as,

$$
\hat{\chi}_{k}(r)=\angle \hat{y}_{b}(r)-\angle \beta(r)-\angle G_{1}\left(\theta_{c}\right) .
$$

Perfect phase synchronization between the JRC enabled radar platform and the communication receiver is required to reach accurate deciphering of symbols.

The communication vector appears only for the direction $\theta_{c}$ and also the receiver needs to have communication vector before the matched filtering operation. Therefore, this makes corresponding operation is directional and secure. However, this communication operation requires coherency between JRC enabled coherent MIMO platform and the receiver. On the other hand, $\theta_{c}$ can be estimated at the communication receiver by calculating the time difference of arrivals (TDoA) of waveforms emitted from first and $M$-th antenna. There are several TDoA techniques in the literature, hence, the details of the TDoA algorithm is out of the scope of this paper.

\subsection{Null-Jitter Dual-Waveform Broadcast}

Since MIMO radar target sensing is not affected by the transmitted waveforms and transmit beampatterns with virtual beamforming at the coherent MIMO radar [16], we can slightly steer the transmit beampattern even if small increase on the null level at the target direction $\theta_{n}$. The progressive phase difference between orthogonal waveforms emitted from the first and the $M$-th antenna caused by steering can be used for information embedding similar as in [10].

Let $\chi_{\Delta_{k}}(r)$ is a phase symbol which is used for the rotation of waveforms emitted from the first and the last antenna element, $\mathbf{w}_{1}$ and $\mathbf{w}_{M}$. Phase symbol $\chi_{\Delta_{k}}(r)$ can be selected from a predefined dictionary $\mathbb{D}_{\Delta}$ when $\log _{2} K$ bits of information is embedded for each pulse.

Let us steer the space-time phase coding matrix as $\theta_{\Delta_{k}} \in$ $\left[-\theta_{\Delta},+\theta_{\Delta}\right)$ which results a progressive phase difference $\chi_{\Delta_{k}}(r)$ between the orthogonal waveforms from $\mathbf{w}_{1}$ to $\mathbf{w}_{M}$,

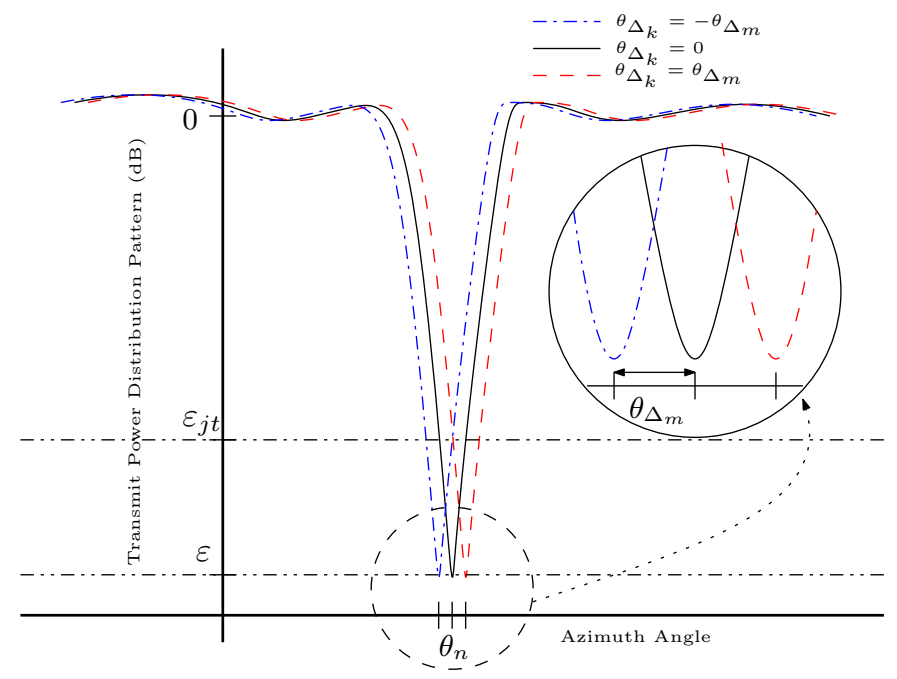

Fig. 3: Transmit patterns when beam steering for Null-Jitter Dual Waveform Broadcast is applied.

$$
\begin{aligned}
\hat{\boldsymbol{\Phi}}_{c}(r) & =\boldsymbol{\Phi} \mathbf{\Upsilon}(r) \\
& =\left[\mathbf{w}_{1}, \mathbf{w}_{2}, \ldots, \mathbf{w}_{M}\right] \operatorname{diag}\{\boldsymbol{v}(r)\} \\
& =\left[\begin{array}{c}
\mathbf{w}_{1} \\
\mathbf{w}_{2} \\
\vdots \\
\mathbf{w}_{M}^{T}
\end{array}\right]\left[\begin{array}{cccc}
1 & 0 & \ldots & 0 \\
0 & e^{-j\left(\chi_{\Delta_{k}}(r) /(M-1)\right)} & \ldots & 0 \\
\vdots & \vdots & \vdots & \vdots \\
0 & 0 & \ldots & e^{-j \chi_{\Delta_{k}}(r)}
\end{array}\right] \\
& {\left[\begin{array}{c}
\mathbf{w}_{1} \\
e^{-j\left(\chi_{\Delta_{k}}(r) /(M-1)\right)} \mathbf{w}_{2} \\
e^{-j\left(2 \chi_{\Delta_{k}}(r) /(M-1)\right)} \mathbf{w}_{3} \\
\vdots \\
e^{-j \chi_{\Delta_{k}}(r)} \mathbf{w}_{M}
\end{array}\right]^{T}=\left[\begin{array}{c}
\boldsymbol{v}(r) \odot \mathbf{u}_{1} \\
\boldsymbol{v}(r) \odot \mathbf{u}_{2} \\
\vdots \\
\boldsymbol{v}(r) \odot \mathbf{u}_{L}
\end{array}\right]=\left[\begin{array}{c}
\overline{\mathbf{u}}_{1} \\
\overline{\mathbf{u}}_{2} \\
\vdots \\
\overline{\mathbf{u}}_{L}
\end{array}\right], }
\end{aligned}
$$

where $\boldsymbol{v}(r)=\left[1, e^{-j\left(\frac{\chi_{\Delta_{k}}(r)}{(M-1)}\right)}, \ldots, e^{-j \chi_{\Delta_{k}}(r)}\right]^{T}, \boldsymbol{\Upsilon}(r)$ is $M \times M$ diagonal matrix of $\boldsymbol{v}(r)$ and $\overline{\mathbf{u}}_{l}$ is the null shifted version of $\mathbf{u}_{l}$. As seen on (37), all the orthogonal waveforms, $\mathbf{w}_{m}$, are only phase rotated and all of the transmit beamforming vectors, $\mathbf{u}_{l}$, are affected by a progressive phase shift vector $\boldsymbol{v}(r)$.

We try to shift the null of $\mathbf{u}_{l}$ as $\theta_{\Delta_{k}}$ via applying the progressive phase shift vector $\boldsymbol{v}(r)$ to $\mathbf{u}_{l}$. Thus, the radiation of $\mathbf{u}_{l}$ towards the $\theta_{n}$ is equal to the radiation of $\overline{\mathbf{u}}_{l}$ towards the direction of $\bar{\theta}_{n}=\theta_{n}+\theta_{\Delta_{k}}$ as in Fig. 3 as,

$$
\begin{aligned}
G_{l}\left(\theta_{n}\right)= & \mathbf{u}_{l}^{*} \mathbf{a}_{t r}\left(\theta_{n}\right)=\bar{G}_{l}\left(\bar{\theta}_{n}\right)=\overline{\mathbf{u}}_{l}^{*} \mathbf{a}_{t r}\left(\theta_{n}+\theta_{\Delta_{k}}\right)=\varepsilon \\
G_{l}\left(\theta_{n}\right)= & {\left[e^{j \varphi_{1}(l)}, e^{j \varphi_{2}(l)}, \ldots, e^{j \varphi_{M}(l)}\right] } \\
& \times\left[1, e^{-j 2 \pi d \sin \left(\theta_{n}\right)}, \ldots, e^{-j 2 \pi(M-1) d \sin \left(\theta_{n}\right)}\right]^{T} \\
= & \sum_{m=1}^{M} e^{j\left[\varphi_{m}-2 \pi(m-1) d \sin \theta_{n}\right]}=\varepsilon
\end{aligned}
$$

$$
\begin{aligned}
\bar{G}_{l}\left(\bar{\theta}_{n}\right)= & {\left[\begin{array}{c}
e^{j \varphi_{1}(l)} \\
e^{j\left[\varphi_{2}(l)-\left(\frac{\chi_{\Delta_{k}}(r)}{(M-1)}\right)\right]} \\
\vdots \\
e^{j\left[\varphi_{M}(l)-\chi_{\Delta_{k}}(r)\right]}
\end{array}\right]^{T} } \\
& \times\left[1, e^{-j 2 \pi d \sin \left(\bar{\theta}_{n}\right)}, \ldots, e^{-j 2 \pi(M-1) d \sin \left(\bar{\theta}_{n}\right)}\right]^{T} \\
= & \sum_{m=1}^{M} e^{j\left[\varphi_{m}-2 \pi(m-1) d\left[\sin \bar{\theta}_{n}+\frac{\chi_{\Delta_{k}}(r)}{2 \pi(M-1) d}\right]\right.}=\varepsilon,
\end{aligned}
$$

and the following equation must hold:

$$
\sin \theta_{n}=\sin \bar{\theta}_{n}+\frac{\chi_{\Delta_{k}}(r)}{2 \pi(M-1) d} .
$$

Then, from (41) the phase symbol $\chi_{\Delta_{k}}(r)$ becomes,

$$
\begin{gathered}
\chi_{\Delta_{k}}(r)=2 \pi(M-1) d\left[\sin \theta_{n}-\sin \bar{\theta}_{n}\right], \text { where } \\
\chi_{\Delta_{k}} \in\left[\mu \pi\left[\sin \theta_{n}-\sin \left(\theta_{n}+\theta_{\Delta}\right)\right],\right. \\
\left.\mu \pi\left[\sin \theta_{n}-\sin \left(\theta_{n}-\theta_{\Delta}\right)\right]\right)
\end{gathered}
$$




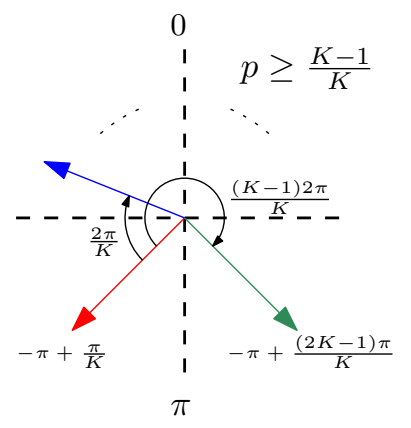

(a)

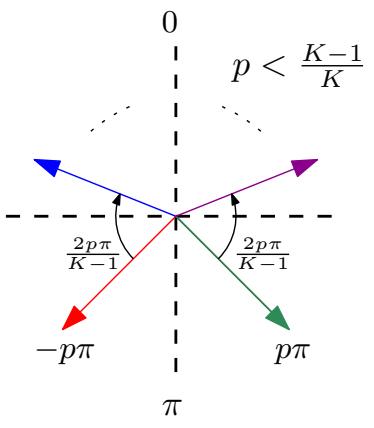

(b)
Fig. 4: M-PSK Symbol Constellation when (a) $p \geq \frac{K-1}{K}$, (b) $p<\frac{K-1}{K}$ for Null-Jitter Dual Waveform Broadcast

and $\theta_{\Delta}$ is a null jitter angle limit which is a positive angle in radian and $\mu$ is a positive constant and equal to $2 d(M-1)$. Then, the phase symbol $\chi_{\Delta_{k}}(r)$ can be selected from a range as,

$$
\begin{aligned}
& \chi_{\Delta_{k}}(r) \in[-p \pi, p \pi), \\
& \text { and } p= \begin{cases}1 & \text { if } f\left(\theta_{n}, \theta_{\Delta_{m}}\right) \geq \frac{1}{\mu} \\
\mu f\left(\theta_{n}, \theta_{\Delta}\right) & \text { if } f\left(\theta_{n}, \theta_{\Delta_{m}}\right)<\frac{1}{\mu}\end{cases}
\end{aligned}
$$

where $p$ is a reducing factor constant in a range $(0,1]$ and $f\left(\theta_{a}, \theta_{b}\right)=\left[\sin \theta_{a}-\sin \left(\theta_{a}-\theta_{b}\right)\right]$. Hence, $\theta_{\Delta_{m}}$ is the maximum steering angle limit which satisfy the condition,

$$
\left\|\mathbf{u}_{l}^{*} \mathbf{a}_{t r}(\theta)\right\| \leq \hat{\varepsilon}_{j t}, \theta \in\left[\theta_{n}-\theta_{\Delta_{m}}, \theta_{n}+\theta_{\Delta_{m}}\right),
$$

for $l=1, \ldots, L$ and where $\hat{\varepsilon}_{j t}$ maximum applicable null level required for the low probability of intercept by the target reconnaissance receiver at $\theta_{n}$. In practice, for small values of $\theta_{\Delta_{m}}$, it is possible to reach closer to the maximum null level $\varepsilon$ in (13).

From (43), when $p \in\left[\frac{K-1}{K}, 1\right]$, the symbol constellation spans full scale as $[-\pi, \pi)$ and traditional M-PSK symbol mapping can be applied in Fig. 4(a). However, if $\theta_{\Delta_{m}}$ is smaller than $1 / \mu$, the communication becomes reduced M-PSK by factor of $p$ similar to the method in [19]. The reduced symbol constellation is given in Fig. 4(b). For larger MIMO arrays, larger distance $d$ or large number of antenna elements $M$, the symbol constellation can easily span to a full scale. Then, the M-PSK dictionary becomes,

$$
\mathbb{D}_{\Delta}=\left\{\begin{array}{ll}
\left\{-\pi+\frac{\pi}{K},-\pi+\frac{3 \pi}{K}, \ldots,-\pi+\frac{(2 K-1) \pi}{K}\right\}, & p \geq \frac{K-1}{K} \\
\left\{-p \pi,-p \pi+\frac{2 p \pi}{K-1}, \ldots, p \pi\right\}, & p<\frac{K-1}{K}
\end{array} .\right.
$$

Using (37), the jitter-wise steered rotated set of orthogonal waveforms can be expressed as,

$$
\mathbf{s}_{\text {com }}(t ; r)=\mathbf{w}(t)^{\Upsilon} \mathbf{Y}(r) .
$$

When the radiated signal reaches to the communication receiver at $\theta_{c}$ which is equipped with omni-directional antenna, the baseband received signal from (11) for $r$-th radar pulse becomes,

$$
\begin{aligned}
y_{b}(t ; r)= & \beta(r) \mathbf{a}_{t r}^{T}\left(\theta_{c}\right) \mathbf{s}_{c o m}(t ; r)+\eta(t ; r) \\
= & \beta(r) \mathbf{a}_{t r}^{T}\left(\theta_{c}\right)\left[\begin{array}{c}
w_{1}(t) \\
e^{-j\left(\chi_{\Delta_{k}}(r) /(M-1)\right)} w_{2}(t) \\
\vdots \\
e^{-j \chi_{\Delta_{k}}(r)} w_{M}(t)
\end{array}\right]^{T} \\
& +\eta(t ; r) .
\end{aligned}
$$

At the receiver, $y_{b}(t ; r)$ is applied to the matched filters of $w_{1}(t)$ and $w_{M}(t)$. Then, the output of the matched filters can be given as,

$$
\begin{aligned}
& \hat{y}_{b}^{1}(r)=\beta(r)\left[1+\sum_{m=2}^{M} e^{-j(m-1) \frac{\chi_{\Delta_{k}}(r)}{(M-1)}} e^{-j 2 \pi(m-1) d \sin \left(\theta_{c}\right)} \xi_{m}^{1}\right] \\
& +\hat{\eta}_{1}(r) \\
& \hat{y}_{b}^{M}(r)=\beta(r)\left[e^{-j \chi_{\Delta_{k}}(r)} e^{-j 2 \pi(M-1) d \sin \left(\theta_{c}\right)}\right] \\
& +\beta(r)\left[\sum_{m=1}^{M-1} e^{-j(m-1) \frac{\chi_{\Delta_{k}}(r)}{(M-1)}} e^{-j 2 \pi(m-1) d \sin \left(\theta_{c}\right)} \xi_{m}^{M}\right]+\hat{\eta}_{M}(r)
\end{aligned}
$$

where $\hat{\eta}_{m}(r)$ is the noise at the output of the $m$-th matched filter whose variance is the same as that of $\eta(t ; r)$ and $\xi_{q}^{m}=$ $\int_{-\infty}^{\infty} w_{q}(\tau) h_{m}(t-\tau) d \tau$ is the $m$-th waveform matched filter output of the $q$-th waveform.

Since, coding waveforms are pseudonoise like signals, any two longer coded waveforms emitted from antenna elements are nearly orthogonal due to their relatively small cross-correlation residues, hence we can write as $\xi_{q}^{m} \approx 0$, when $m \neq q$. Then, the output of the matched filters becomes,

$$
\begin{gathered}
\hat{y}_{b}^{1}(r)=\beta(r)+\hat{\eta}_{1}(r) \\
\hat{y}_{b}^{M}(r)=\beta(r) e^{-j \chi_{\Delta_{k}}(r)} e^{-j 2 \pi(M-1) d \sin \left(\theta_{c}\right)}+\hat{\eta}_{M}(r) .
\end{gathered}
$$

To detect the embedded communication symbols, the receiver estimates the phase difference between the output of the matched filter of $w_{1}(t)$ in (50) and $w_{M}(t)$ in (51) as,

$$
\hat{\chi}_{k}(r)=\operatorname{angle}\left(\frac{\hat{y}_{b}^{1}(r)}{\hat{y}_{b}^{M}(r)}\right)-2 \pi(M-1) d \sin \left(\theta_{c}\right) .
$$

Although this method does not require coherency between the receiver and transmit MIMO array, the communication receiver needs to knows its direction $\theta_{c}$ with respect to the coherent MIMO radar transmit array and also, it must have a perfect knowledge of the displacement between first to last element in the transmit array $(M-1) d$. Thus, the communication receiver is able to remove the phase term $2 \pi(M-1) d \sin \left(\theta_{c}\right)$ while detecting the embedded phase symbol $\chi_{\Delta_{k}}$.

\subsubsection{Off-line Symbol Constellation Reconstruction}

If the radar waveform code length is not long enough, crosscorrelation residues may increase to a significant level which may affect the symbol constellation. In that case, removing the 


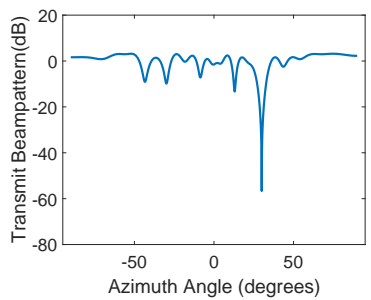

(a)

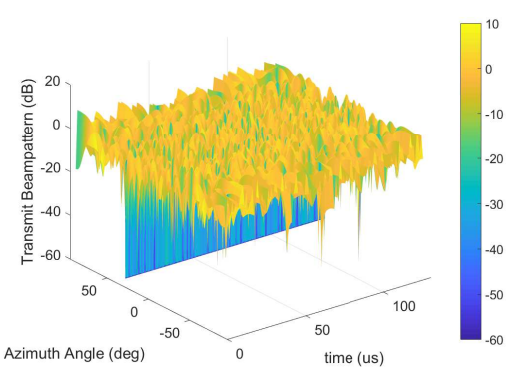

(e)

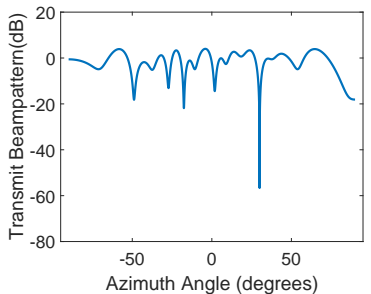

(b)

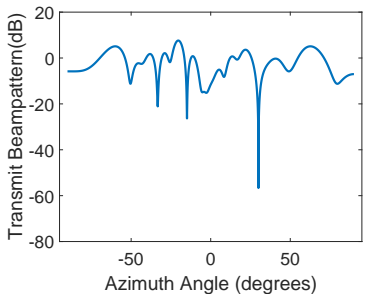

(c)

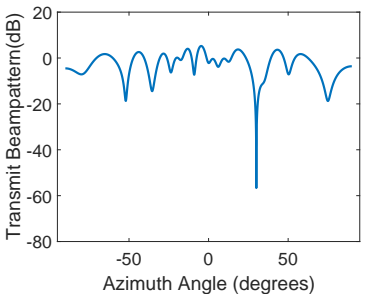

(d)

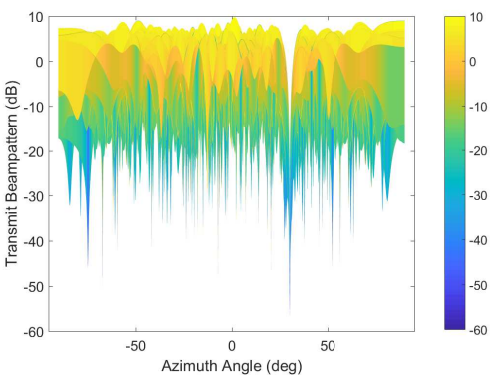

(f)

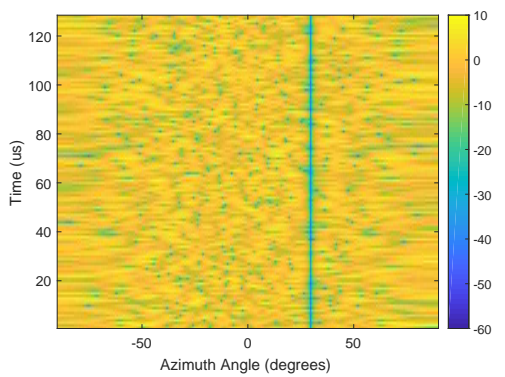

(g)

Fig. 5: Transmit beampatterns of space-time phase coding waveform designed with technique in [16] with null at $\theta_{n}=30^{\circ}$ during time period of (a) first subpulse; (b) second subpulse; (c) third subpulse; and (d) fourth subpulse and (e,f,g) whole radar pulse.

phase term $2 \pi(M-1) d \sin \left(\theta_{c}\right)$ in (52) will not suffice to retrieve symbols correctly. At this point, we have to find a way to calculate new distorted symbol constellation.

Let's assume that the receiver knows all transmitted MIMO radar waveforms, communication direction $\theta_{c}$ and phase symbol dictionary, then the new phase symbol constellation can be estimated via preprocessing. This preprocessing can be done by matched filtering the steered modulated signal $\mathbf{s}_{\text {com }}(t)$, which is $y_{c r}(t)$, in off-line manner. The resulting signal $y_{c r}(t)$ can be given as,

$$
\begin{aligned}
y_{c r}\left(t ; \chi_{c} ; \theta_{c}\right) & =\mathbf{a}_{t r}^{T}\left(\theta_{c}\right) \mathbf{s}_{c o m}(t ; r) \\
& =\mathbf{a}_{t r}^{T}\left(\theta_{c}\right)\left[\begin{array}{c}
w_{1}(t) \\
e^{-j\left(\chi_{c} /(M-1)\right)} w_{2}(t) \\
\vdots \\
e^{-j \chi_{c}} w_{M}(t)
\end{array}\right]^{T},
\end{aligned}
$$

where $\chi_{c}=\left\{\chi_{c}^{1}, \ldots, \chi_{c}^{K}\right\} \in \mathbb{D}_{\Delta}$. Then, using the matched filter response of $y_{c r}(t)$ for each symbol in the dictionary, new phase dictionary $\overline{\mathbb{D}}_{\Delta}$ for each communication direction can be given as,

$$
\overline{\mathbb{D}}_{\Delta}\left(\theta_{c}\right)=\left\{\operatorname{angle}\left(\frac{\hat{y}_{c r}^{M}\left(\chi_{c}^{1} ; \theta_{c}\right)}{\hat{y}_{c r}^{1}\left(\chi_{c}^{1} ; \theta_{c}\right)}\right), \ldots, \text { angle }\left(\frac{\hat{y}_{c r}^{M}\left(\chi_{c}^{K} ; \theta_{c}\right)}{\hat{y}_{c r}^{1}\left(\chi_{c}^{K} ; \theta_{c}\right)}\right)\right\},
$$

where $\hat{y}_{c r}^{m}\left(\chi_{c}^{k} ; \theta_{c}\right)$ is the output of the $m$-th waveform matched filter of the signal $y_{c r}\left(t ; \chi_{c} ; \theta_{c}\right)$. Then, the embedded binary sequence can be deciphered according to the new phase dictionary $\overline{\mathbb{D}}_{\Delta}\left(\theta_{c}\right)$.

\section{Performance Evaluation}

In this section, simulation results are presented evaluating the space-time waveform coding and orthogonality performance for proposed methods in Sec. 3. Then, the communication performance of the proposed methods detailed in Sec. 4 are investigated in terms of bit error rate (BER).

A uniform linear transmit array with 16 antennas spaced with 0.57 of a wavelength is used at the simulations. During each radar pulse, information bits are transmitted towards the communication direction $\theta_{c}$. For all evaluations, the communication direction is selected as $\theta_{c}=-22^{\circ}$, the null direction is selected as $\theta_{n}=30^{\circ}$ and for each test, a sequence of $10^{4}$ radar pulses were transmitted to the channel, unless otherwise stated. In order to reach results, a Monte Carlo approach has been used over 100 tests and all simulations are done in MATLAB.

\subsection{Space-Time Waveform Coding Performance}

We first study the performance of the proposed methods in terms of level of fulfillment of the requirements about the transmit beampattern, waveform orthogonality and total number of permutation iteration needed to form all orthogonal waveforms. Simulations are run for 128 subpulses and 16 antenna elements.

\subsubsection{Transmit Beampattern}

The proposed waveform design approaches for enabling JRC are implemented based on a coherent MIMO radar in Fig. 5. Using the GA based transmit beamforming technique explained in Sec. 3.1, we reach the first transmit beamforming vector $\mathbf{u}_{1}$, whose transmit beampattern is given in Fig. 5(a). Fig. 5(a) shows the transmit beampattern of the designed waveform with a design requirement of having a null at the broadside angle of $30^{\circ}$. The null depth at the transmit beampatterns generated by 


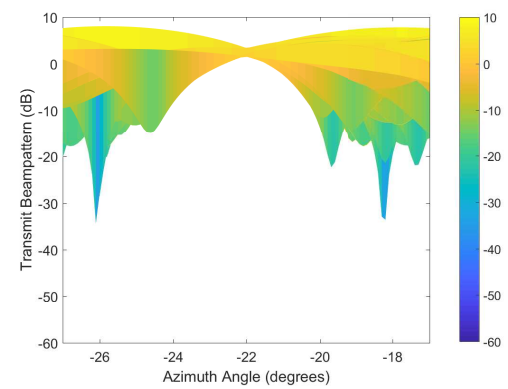

(a)

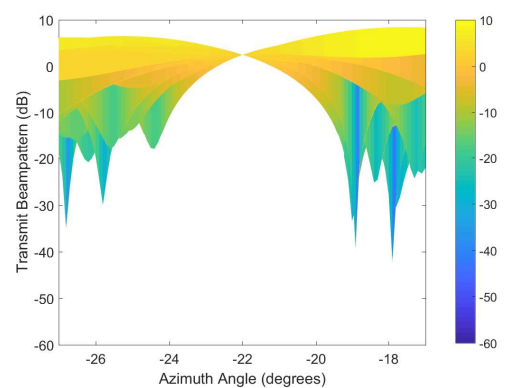

(d)

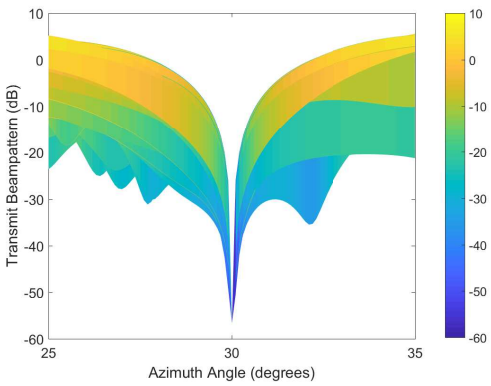

(b)

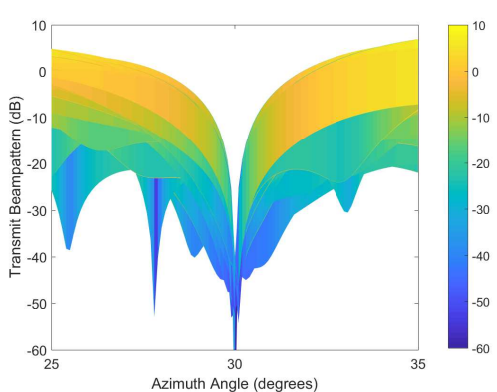

(e)

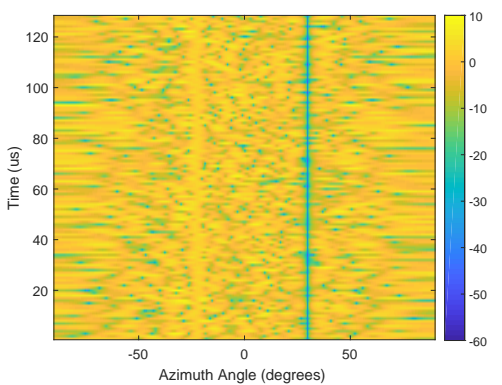

(c)

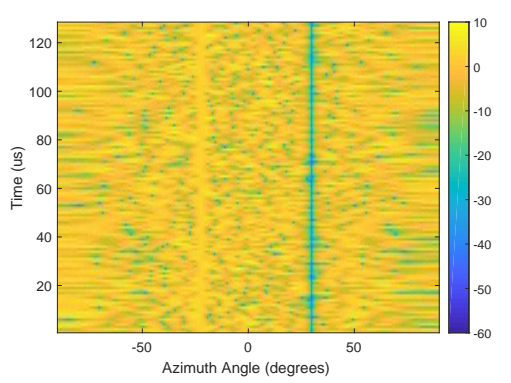

(f)

Fig. 6: $(\mathrm{a}, \mathrm{b}, \mathrm{c})$ is the transmit beampatterns of space-time coding waveforms designed with the null direction fixed waveform coding when $\Delta=1 \mathrm{~dB}$ and (d,e,f) with the communication direction fixed waveform coding when $\hat{\varepsilon}=-40 \mathrm{~dB}$ at angles $\theta_{c}=-22^{\circ}$ and $\theta_{n}=30^{\circ}$.

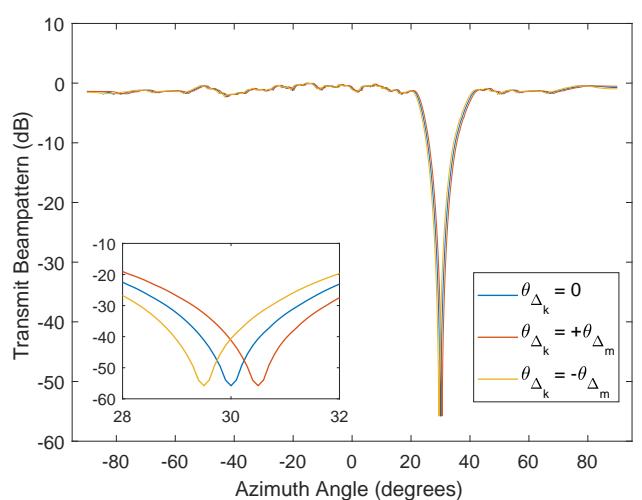

Fig. 7: Average transmit beampattern for null-jitter dual-waveform broadcast method applied when $\varepsilon_{j t}=-40 \mathrm{~dB}, \theta_{\Delta_{m}}=0.5$ and $\theta_{n}=30$ degrees

the technique in [16] is about $55 \mathrm{~dB}$ for each subpulses as in Fig. 5. Hence, with the designed transmit beamforming vectors MIMO radar antenna radiates almost no power in the null direction $\theta_{n}$.

All 128 transmit beampatterns of the space-time coding waveforms designed with the technique in [16] is shown in Figures $5(\mathrm{e})$ to $5(\mathrm{~g})$. We can easily see that the null requirement is preserved during the whole radar pulse duration.

With the proposed techniques in Sec. 3.2 and Sec. 3.3, the space-time coding waveforms are redesigned and their transmit beampatterns are shown in Fig. 6, when the communication angle is selected as $\theta_{c}=-22^{\circ}$. For null direction fixed beamforming technique, communication distortion factor is selected as $\Delta=1 \mathrm{~dB}$. Then, it can be noticed from the Fig. 6(b), there is a small variation on the radiation towards the communication direction, $\theta_{c}=-22^{\circ}$. On the other hand, using the communication direction fixed beamforming technique the radiation level is exactly preserved during the whole radar pulse in Fig. 6(e). However, null depth varies below $-40 d B$, when the relaxed null level $\hat{\varepsilon}$ is selected as $-40 d B$.

Moreover, the average transmit beampattern is shown in Fig. 7 for null-jitter dual-waveform broadcast method is applied with the parameters $\varepsilon_{j t}=-40 \mathrm{~dB}, \theta_{\Delta_{m}}$ is $0.5^{\circ}$ and $\theta_{n}=30^{\circ}$. From the figure, at least $-40 \mathrm{~dB}$ null level is preserved for the time average over the radar pulse width $T_{P W}$.

\subsubsection{Waveform Orthogonality}

In our work, the effect on the radar processing performance of the proposed waveform generation techniques detailed in Sec. 3 is analyzed in this section by inspecting the normalized virtual beampatterns of generated waveforms using the VAB technique in [18].

While satisfying a deep null at angle $\theta_{n}=30^{\circ}$ towards broadside angle as a transmit beamforming requirement, emitted waveforms from MIMO antenna elements needs to be orthogonal in the time domain. Therefore, the orthogonality level of the proposed techniques are revealed via three different ways. First, the auto and cross correlation functions of the designed waveforms and then their normalized covariances are examined. Lastly, virtual beampatterns formed with the technique detailed in [18] using the virtual beamforming filter are investigated.

Auto and cross-correlations between MIMO radar wave- 

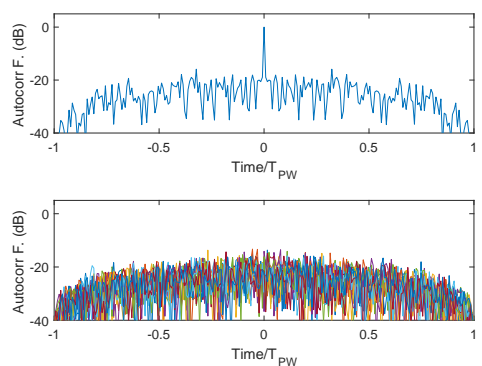

(a)

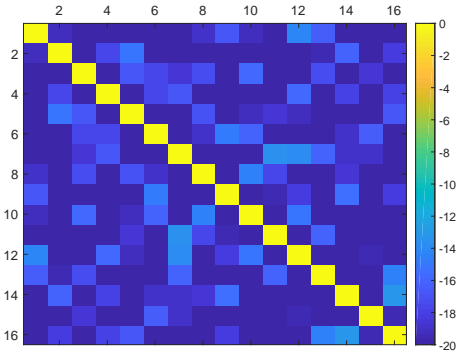

(d)
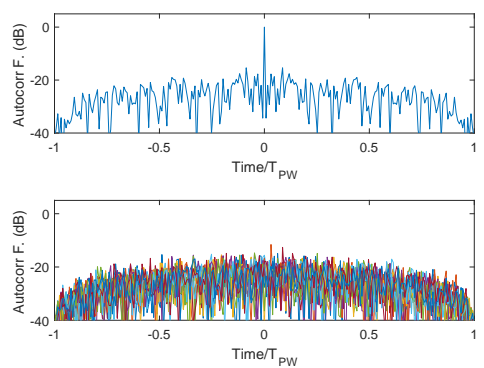

(b)

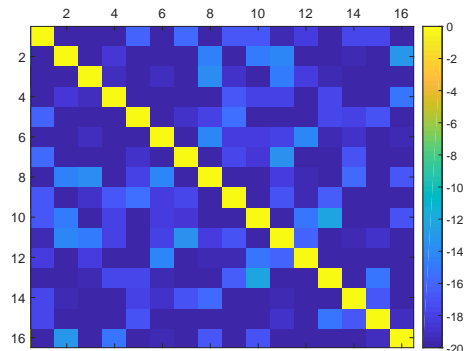

(e)
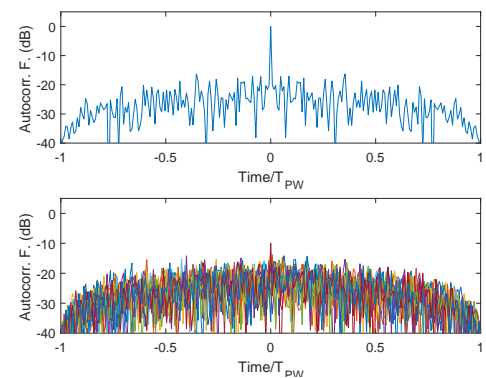

(c)

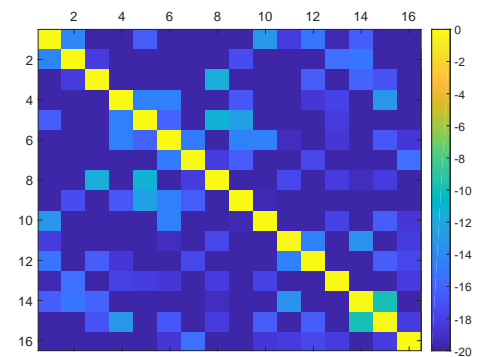

(f)

Fig. 8: Auto\&Cross-Correlations between waveforms emitted from 1st to the other antenna elements (a) for the technique in [16], (b) for the proposed technique in Sec. 3.2, (c) from 14th to the other antenna elements for the proposed technique in Sec. 3.3. Covariances between waveforms emitted for the technique (d) in [16], (e) in Sec. 3.2, (f) in Sec. 3.3

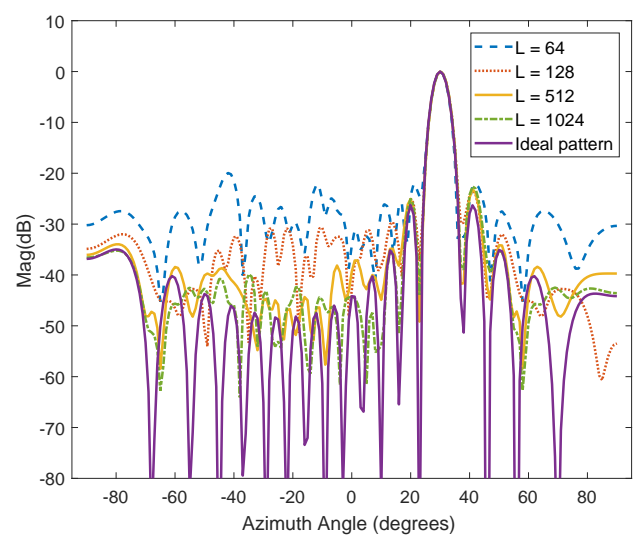

Fig. 9: Normalized virtual beam formed at MIMO radar receiver using spacetime coding waveforms for different code length when $\theta_{n}=30$

forms emitted from 1-st to the other antenna elements for the technique in [16] is shown in Fig. 8(a). The sidelobes are around the level of $1 / \sqrt{L}$ which is also typical for the pseudorandom waveforms. Besides, the two designed waveforms are almost orthogonal due to their relatively small cross-correlation residues at any time as in Fig. 8(a) and the cross-correlation function of the designed waveform pairs for any antenna element is also almost under the level of $1 / \sqrt{128}$ which is around $-20 \mathrm{~dB}$. For the proposed method in Sec. 3.2, the designed waveforms show the same correlation behavior as the technique in [16] in Fig. 8(b), although the proposed method offers almost a stable communication channel to a specific direction.
On the other hand, the proposed method in Sec. 3.3 displays a small degradation by means of orthogonality when inspecting the cross-correlations between waveforms emitted from 14-th and 15-th elements as in Fig. 8(c). Besides, these results can be easily seen on the $\mathrm{dB}$ scale images of the covariance matrix of all waveforms in Figures 8(d) to 8(f).

Lastly, we investigate the orthogonality conditions of the designed waveforms by inspecting the normalized virtual beampatterns. The code length is selected 128 and 16 antenna elements are considered in simulations. The target is expected at the broadside direction $30^{\circ}$ which is also the same as the null direction $\theta_{n}$. In each figure, we compare resulted beampatterns with an ideal pattern which is generated from perfectly orthogonal condition. In Fig. 9, waveforms with longer code lengths exposes lower sidelobes due to smaller effect of their crosscorrelations residues at the matched filters. Moreover, virtual beampattern for varying pre-distortion level $\Delta$ with the condition in (18) in null direction fixed technique is shown in Fig. 10(a). Beampatterns synthesized by the waveforms with different pre-distortion levels display almost the same maximum sidelobe levels. This shows that the condition in (18) does not ruin the orthogonality between waveforms. Likewise, beampatterns with different relaxed null level constant $\hat{\varepsilon}$ with the condition (22) in communication direction fixed technique expose almost the same maximum sidelobe levels. On the other hand, bigger sidelobes can be seen from the Fig. 10(c). The condition (44) clearly affects the orthogonality of the waveforms for higher steering angle $\theta_{\Delta}$. For the null jitter dual-waveform broadcast method which is detailed in Sec. 4.3, larger jitter provides higher information symbol span at the constellation, 


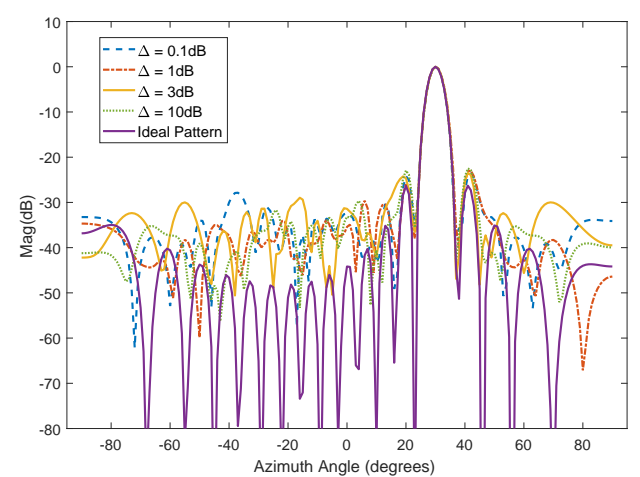

(a)

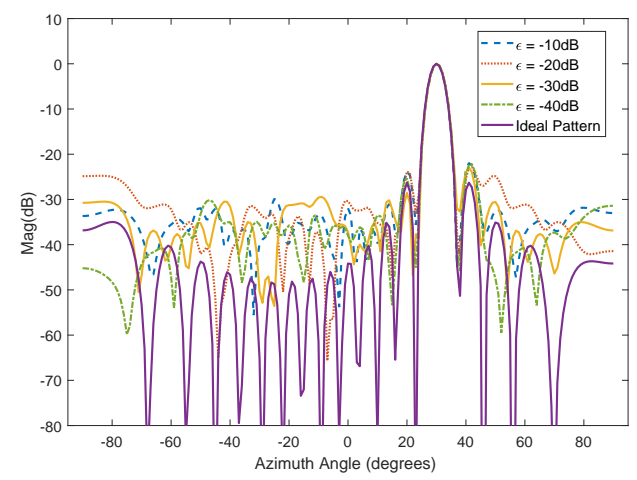

(b)

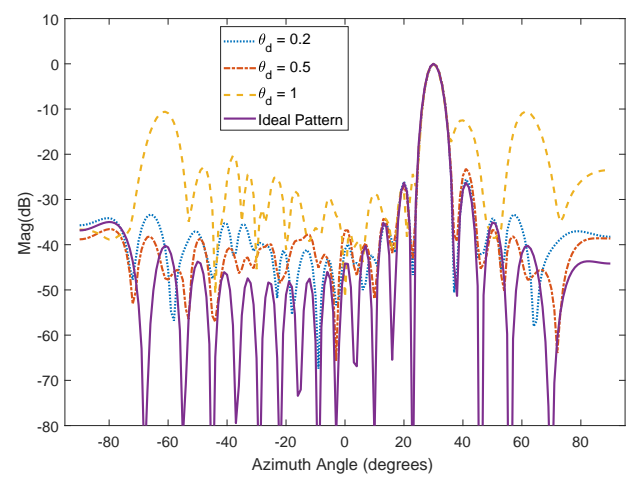

(c)

Fig. 10: Normalized virtual beam formed at MIMO radar receiver using spacetime coding waveforms designed (a) for varying pre-distortion level $\Delta$ with the condition in (18) in null direction fixed technique, (b) for varying relaxed null level constant $\hat{\varepsilon}$ with the condition (22) in communication direction fixed technique and (c) for varying steering angle $\theta_{\Delta}$ with condition (44), when $\theta_{n}=$ 30 .

however the jitter angle has to be small enough not to disturb radar operation by ruining the waveform orthogonality.

\subsubsection{Total Number of Iteration}

Although the waveform generation process we have proposed is an offline process, in this part, we investigate the total permutation iterations needed to form orthogonal radar waveforms. In the process, the code length is selected 128 and 16 antenna elements are considered. The target is expected at the

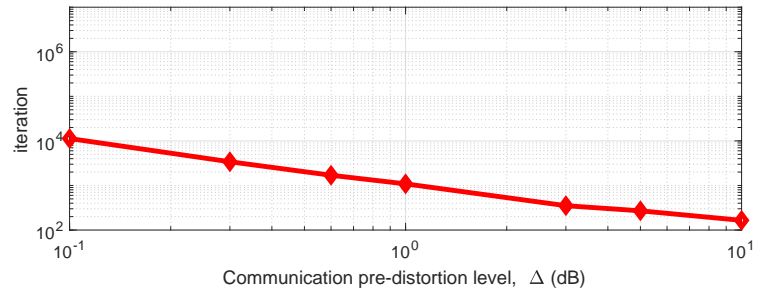

(a)

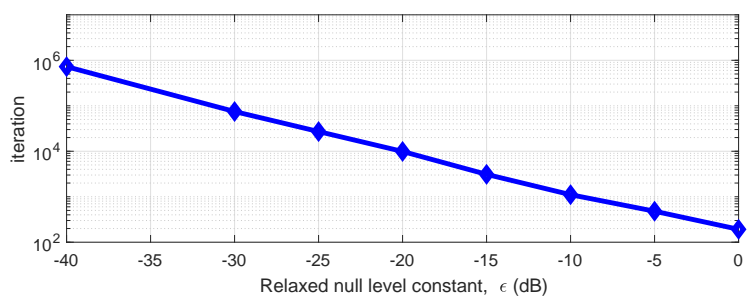

(b)

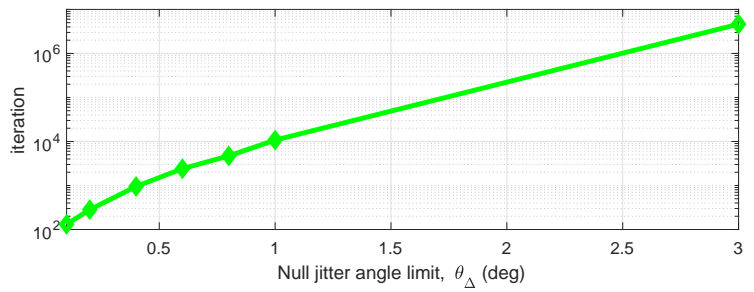

(c)

Fig. 11: Total iterations for the proposed methods for varying (a) communication pre-distortion level $\Delta$ with the condition in (18) in null direction fixed technique, (b) relaxed null level constant $\hat{\epsilon}$ with the condition (22) in communication direction fixed technique and (c) for varying null jitter angle limit $\theta_{\Delta}$ with condition (44), when $M=16, L=128, \theta_{c}=-22^{\circ}$ and $\theta_{n}=30^{\circ}$.

broadside direction $30^{\circ}$, i.e. the null direction $\theta_{n}=30^{\circ}$, when the communication angle is selected as $\theta_{c}=-22^{\circ}$. Using the first transmit beamforming vector $\mathbf{u}_{1}$, whose transmit beampattern is given in Fig. 5(a), proposed waveform generation techniques are applied with varying constraints.

Number of iterations depend entirely on strictness of the corresponding constraints inside the proposed methods. Total iterations for the varying constraints are given in Fig.11. From the figures, it is clear that iteration count exponentially increased when the constraint is linearly increased or decreased. Total iterations for varying communication pre-distortion level $\Delta$ with the condition in (18) in null direction fixed technique is investigated in Fig. 11(a). In order to reach $1 \mathrm{~dB}$ pre-distortion level, almost 1000 permutations needed to form all orthogonal waveforms. In Fig. 11(b), total iterations for varying relaxed null level constant $\hat{\epsilon}$ with the condition (22) in communication direction fixed technique is displayed. A -30dB null level, which may be acceptable for most practical applications, can be achieved for almost 70000 permutation iterations. It should be noted that, $-30 \mathrm{~dB}$ relaxed null level constant secures that there are no other transmit beampattern above this constraint, hence the emitted average transmit power towards the target direction must be several $\mathrm{dB}$ below. Lastly, total iterations for 
varying null jitter angle limit $\theta_{\Delta}$ with condition (44) is shown in Fig. 11(c). Selecting a small null jitter angle limit provides small sidelobe levels at the normalized virtual beam formed at MIMO radar receiver from the Fig. 10(c). On the other hand, higher null jitter angle grants more symbol span for the communication. Considering all of these issues, a $0.5^{\circ}$ null jitter angle limit, which is acceptable by looking in terms of orthogonality and communication symbol span, can be obtained for almost 1500 iterations from the in Fig. 11(c).

If the number of antennas $M$ is small relative to the code length, or the constraint is so stringent, it is possible to reach no solution case or it may take so many iteration to form all radar waveforms. For no solution case, besides repeating the procedure with relaxed constraints, the base transmit beamforming vector $\mathbf{u}_{1}$ can also be redesigned using GA algorithm, and, then the procedure can be repeated with the new $\mathbf{u}_{1}$. If the number of antennas $M$ is large, that it becomes less and less probable to reach no solution case. Although it may take long iterations when stringent constraint is selected, the waveform generation procedure is an offline process. All calculations should be done before the JRC operation. Lastly, it is worth mentioning that the permutation of $M$ number of phase variables, $\varphi_{m}(l)$, means randomly picking a solution from $M$ ! different solutions. In our simulations, $M$ is selected as 16 , hence we can apply our constraints to a huge solution set which contains approximately $2.09 \times 10^{13}$ number of solutions.

\subsection{BER Performance}

The performance of the proposed information embedding techniques are evaluated via Monte-Carlo simulations with 100 trials. Each trial contains $10^{4}$ radar pulses and totally $10^{6}$ pulses are used for investigation by BER. Simulations are run for 128 sub-pulses and 16 antenna elements, unless otherwise stated.

\subsubsection{Intra-Pulse Directional Communication}

In this communication method, each radar sub-pulse is modulated via differential M-PSK technique without violating the space-time orthogonality between MIMO waveforms and transmit beamforming constraints. First, we try to understand the effect of the pre-distortion level $\Delta$. Then, using the waveforms which are synthesized under different pre-distortion levels are used in simulations when 4-DPSK is applied. The results are given in Fig. 12(a). $\Delta=0 \mathrm{~dB}$ indicates that the related waveforms are produced by communication direction fixed method in Sec. 3.3. Hence, communication direction fixed method shows slightly better BER performance as we expected, thus unexpected signal variations at the receiver results performance degradation like a fading effect. The curves up to $1 \mathrm{~dB}$ display close behavior as $0 \mathrm{~dB}$. Besides, $3 \mathrm{~dB}$ pre-distortion level curve shows only $2 \mathrm{~dB}$ SNR degradation which is also acceptable. However, higher level curves totally break down the BER performance.

Fig. 12(b) illustrates the BER performance with different DPSK techniques when communication pre-distortion level is $3 d B$. Although 64-DPSK method performance requires high SNR levels, 2 and 4 symbol DPSK show good performance at

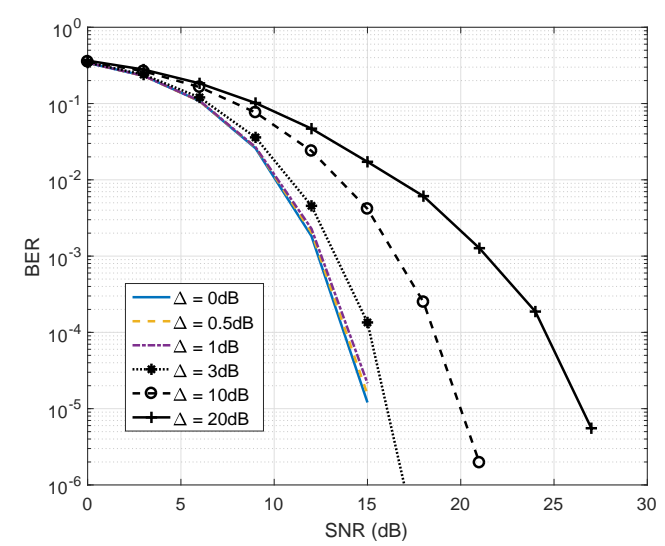

(a)

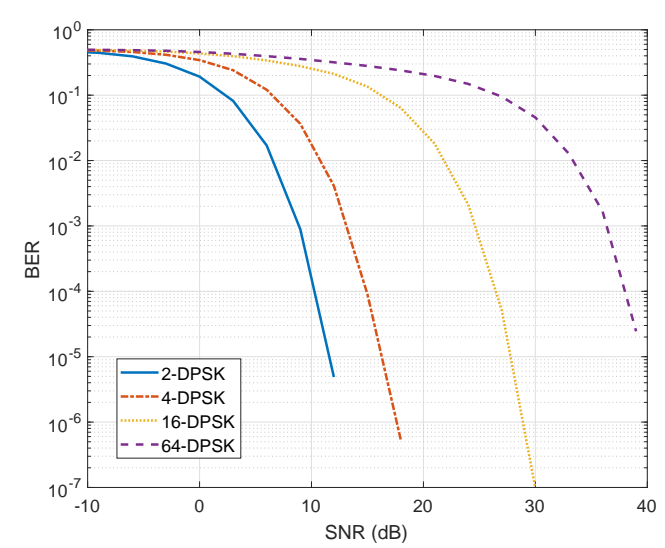

(b)

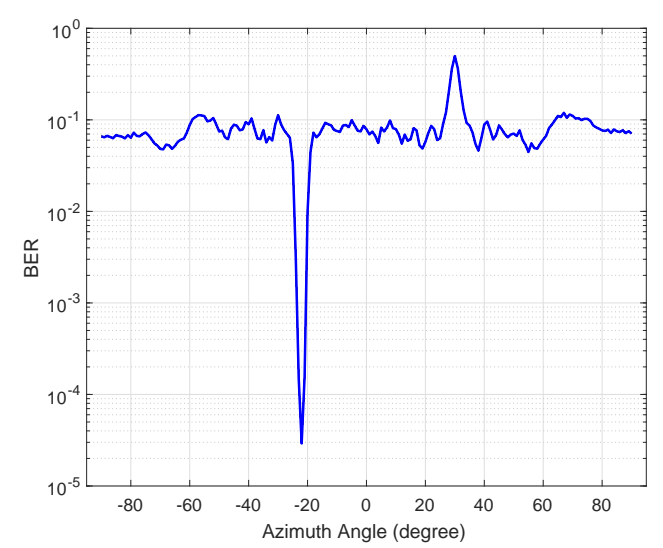

(c)

Fig. 12: Bit error rate of Intra-Pulse Directional Communication technique for different SNR levels, (a) for varying pre-distortion level $\Delta$ when 4-DPSK is applied, (b) for different DPSK methods when $\Delta=3 d B$ and (c) for varying broadside angle when $\theta_{c}=-22^{\circ}$.

lower SNR levels. Moreover, directivity of the proposed communication method is investigated in Fig. 12(c). The results are displayed for varying receiver angle when the communication is fixed to $-22^{\circ}$. The communication symbols can be deciphered only from the specific direction $\theta_{c}=-22^{\circ}$. 


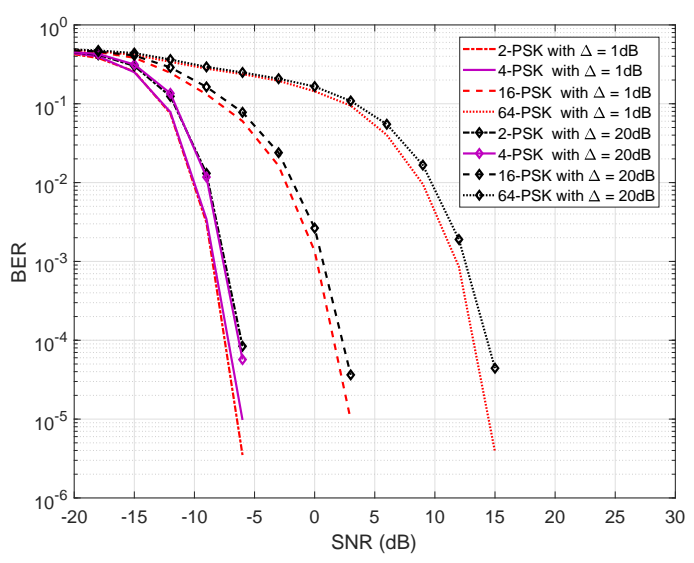

(a)

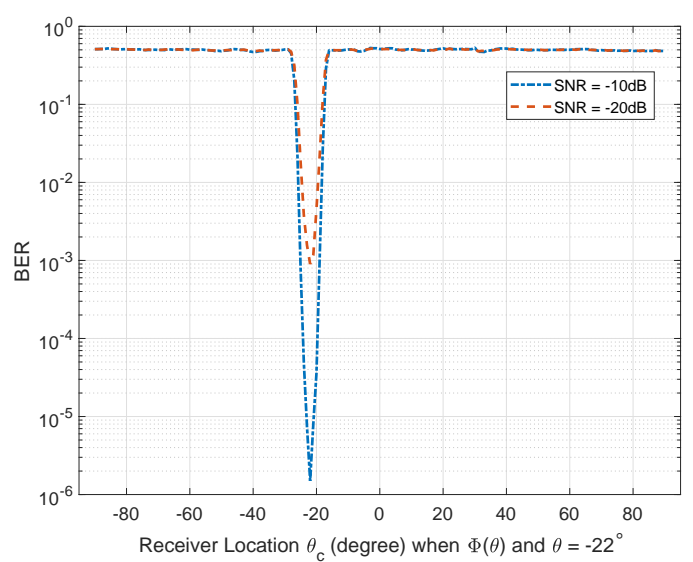

(b)

Fig. 13: Bit error rate of Code-Driven Secure Communication technique, (a) for different MPSK methods and different SNR levels, (b) for varying communication direction $\theta_{c}$ when the code fixed at azimuth angle $-22^{\circ}$ and fixed to $\theta_{c}$ angle $(\mathrm{SNR}=-8 \mathrm{~dB})$.

\subsubsection{Code-Driven Secure Communication}

This method encodes coherent MIMO radiation pattern via an information vector during each radar pulse. Then, the phase of the encoded vector is rotated pulse by pulse. For the simulations, 4 level polyphase pseudo-random coded information vector is selected and it is assumed that this vector is exactly known by the receiver. First, the performance of the proposed method for various SNR levels and different phase rotation modulation techniques are investigated. Proposed method shows outstanding BER performance compared to other proposed method. Hence, the information is carried via a code length of 128 , thus, this method has a coding gain of almost 20dB. Moreover, the waveforms are generated with null direction fixed method in Sec. 3.2 for two pre-distortion level $\Delta .1 \mathrm{~dB}$ and $20 \mathrm{~dB}$ predistortion levels are compared in terms of BER in Fig. 13(a). Only $1 \mathrm{~dB}$ performance improvement is noticed from the figure. Hence, the reason is that the average power over the whole radar pulse is almost the same for both pre-distortion levels.

Fig. 13(b) illustrates the directivity of the communication technique. When the space-time code matrix $\Phi$ is synthesized only for the communication angle $-22^{\circ}$, the communication becomes invisible for other directions, although the receivers at different angles perfectly know the information vector. On the other hand, only exception is that if the communication direction was the same as the null direction, the communication would not be possible due to the low average power received at that direction.

\subsubsection{Null-Jitter Dual-Waveform Broadcast}

Null-Jitter broadcast method uses the progressive phase difference between orthogonal waveforms radiated from the antenna elements to embed information. This progressive phase difference results a small jitter on transmit beamforming.

First, BER performance of the different PSK methods with and without applying off-line correction process detailed in Sec. 4.3.1 are examined in Fig. 14(a), when the code length is fixed at 512. Corrected 2-PSK outperforms all other methods due to its larger symbol distance. Off-line correction process provides significant gain especially for 16-PSK at higher SNR levels. BER performance for different radar waveform code length is inspected for various SNR levels in Fig. 14(b). Although, $6 \mathrm{~dB}$ separation is expected between curves from the coding gain, slightly more separation can be observed. Waveform orthogonality can also be enhanced with increasing code length. Unlike other proposed methods, in null-jitter method two radar waveforms are used for transmitting information symbols in a tandem manner. Therefore, the receiver needs to know and use these two orthogonal radar waveforms in their matched filters. In this case, waveform orthogonality also affects the communication performance. Therefore, When 4-PSK method is used for communication, higher code length displays better performance as expected.

Moreover, the effect of the null jitter angle limit $\theta_{\Delta}$ on communication performance is investigated when the code length is selected as 512. Fig. 14(c) illustrates the BER under different $\theta_{\Delta}$ for various SNR levels. It should be noted that, small null jitter angle presents small information symbol span for communication. In other words, small null jitter angle prevents information symbols span on a full scale phase constellation. Therefore, small $\theta_{\Delta}$ angles show poor BER performance. From the equation in (42), minimum null jitter angle required for full span symbol constellation becomes $3.4^{\circ}$ for $M=16$ and $d=0.5$. Highest null jitter angle can be selected for best performance on BER, however, $\theta_{\Delta}$ angle must be small enough to still maintain LPI at null direction $\theta_{n}$. For this particular example, $\theta_{\Delta}$ angle must be $0.5^{\circ}$ to reach maximum applicable null limit $-40 \mathrm{~dB}$ as in Fig. 7.

For the ideal case, the approximation $\xi_{q}^{m} \approx 0, m \neq q$ detailed in (50) must be hold for longer coded waveforms. However, small cross correlation residues at the receiver matched filter output disturb symbol constellation as a function of communication direction $\theta_{c}$ as in Fig. 15(a). When null jitter angle limit is selected as $\theta_{\Delta}=0.5^{\circ}$ for 4-PSK communication method, the ideal minimum symbol span has to be fixed to $2 p \pi /(K-1)$ which is equal to $15.5^{\circ}$. On the other hand, in reality, symbol span fluctuates around the ideal symbol span due to the crosscorrelation residues between radar waveforms. For higher code 


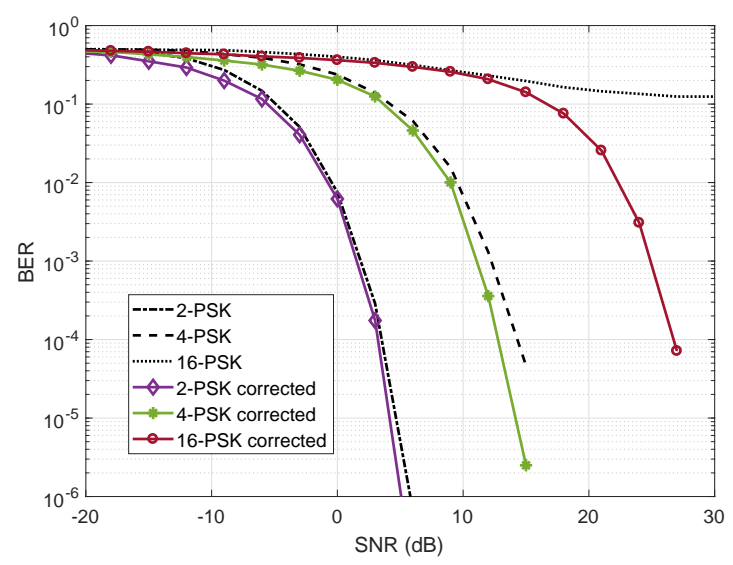

(a)

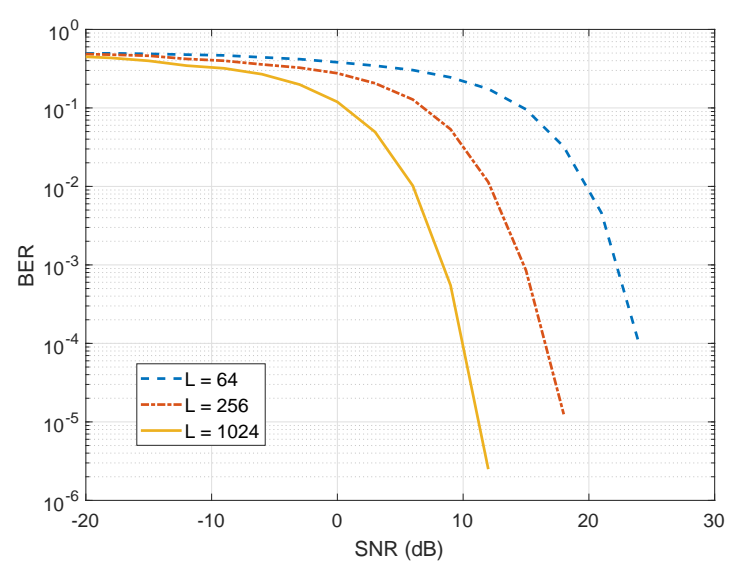

(b)

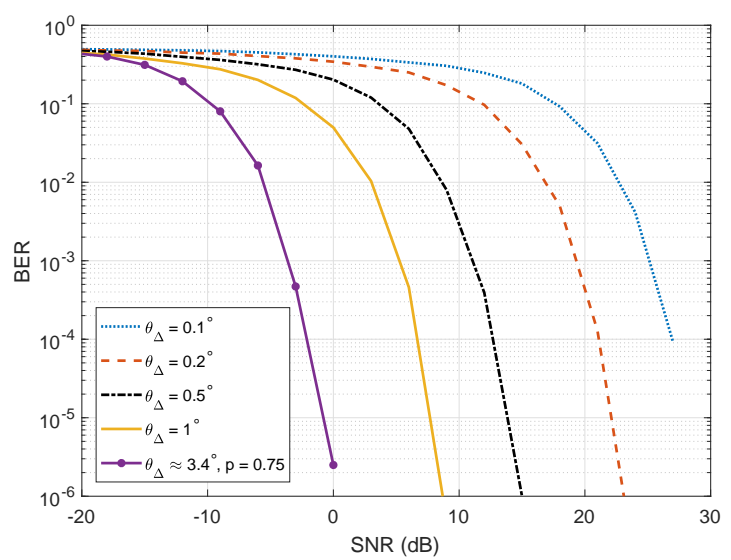

(c)

Fig. 14: Bit error rate of Null-Jitter Dual-Waveform Broadcast technique, (a) for different MPSK methods, different code lengths, (b) for different null jitter angle limit $\theta_{\Delta}$ while varying SNR levels. (c) Minimum symbol span in degrees with different code lengths for varying communication direction $\theta_{c}$.

length this fluctuation decreases, however not vanishes. Using the correction method detailed in Sec. 4.3.1, BER is calculated for all communication directions as in Fig. 15(b). Also, Fig. 15(b) illustrates the broadcast behaviour of the proposed tech-

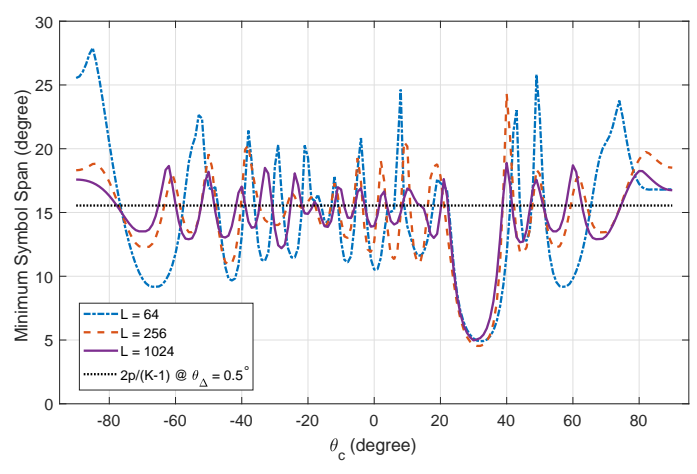

(a)

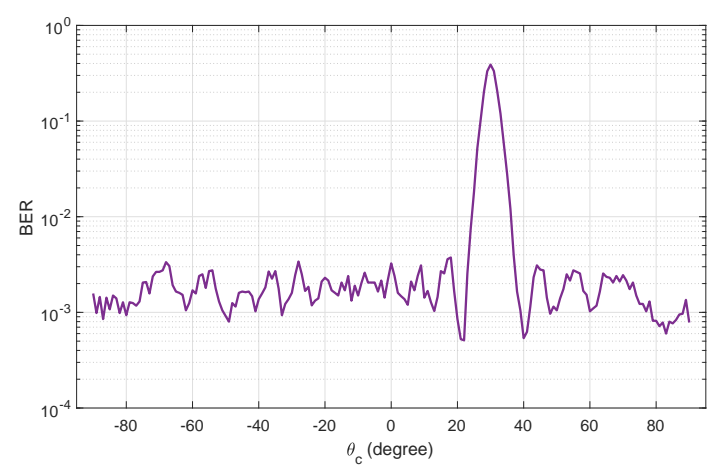

(b)

Fig. 15: Minimum symbol span (a) in degrees with different code lengths and BER (b) for varying communication direction $\theta_{c}$ when $\theta_{n}=30^{\circ}$

nique. Except the null direction at $30^{\circ}$, almost the same bit error rate for all directions can be provided with the proposed technique.

Fig. 16 illustrates normalized symbol constellations of parameters $\chi_{\Delta_{k}}$ in (42), $\hat{y}_{b}^{1}$ in (50), $\hat{y}_{b}^{M}$ in (51), $\hat{y}_{b}^{M} / \hat{y}_{b}^{1}$ and $\hat{\chi}_{k}$ in (52) for different cases under 10dB SNR level. First diagram is the information symbol intended to transmit, $\chi_{\Delta_{k}}$. Then, second and third diagrams show matched filter outputs of the 1-st and $M$-th waveforms, $\hat{y}_{b}^{1}$ in (50), $\hat{y}_{b}^{M}$ in (51). Fourth diagram shows the relative phase and last depicts relative phase of the dual waveforms, $\hat{\chi}_{k}$ in (52), after removing the phase term coming from communication direction, $2 \pi(M-1) d \sin \left(\theta_{c}\right)$. For the first and the second cases (rows in figures) at the simulations in Fig. 16(a) and Fig. 16(b), radar waveforms with code length 1024 are used for communication to angle $60^{\circ}$ and $-55^{\circ}$, respectively and null jitter angle is selected as $1^{\circ}$. When the last symbol diagrams of the first two cases are investigated, two different symbol span can be noticed. Communication at $-55^{\circ}$ spans less angle space. Moreover, the effect of the radar waveforms code lengths with 1024 and 128 on the symbol constellation are shown in Fig. 16(c) and Fig. 16(d). Although, both of the case runs on 10dB SNR level, constellation with lower code length has more dispersive behaviour. 

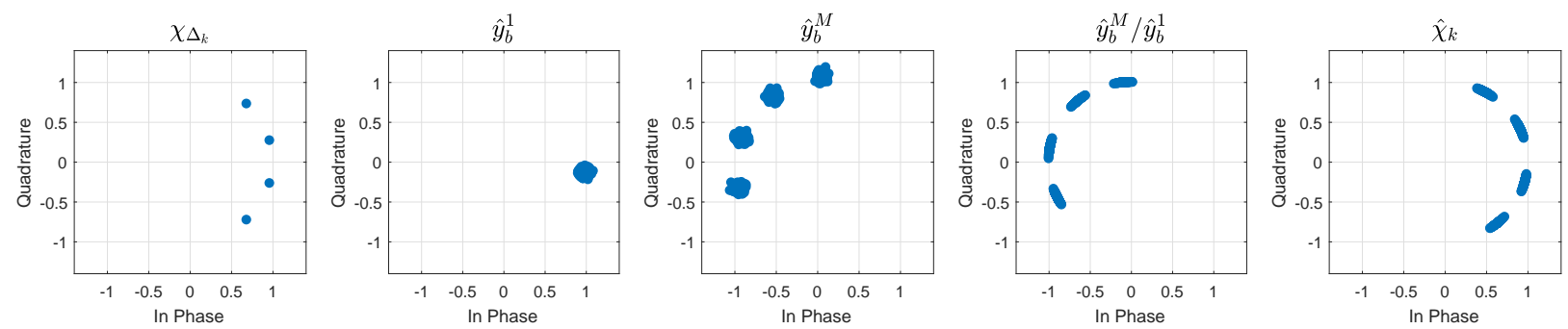

(a)
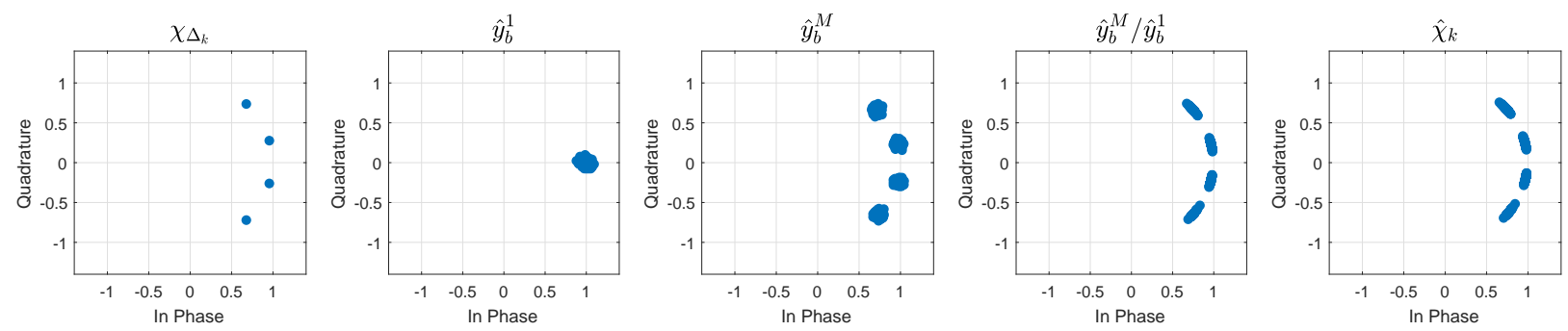

(b)
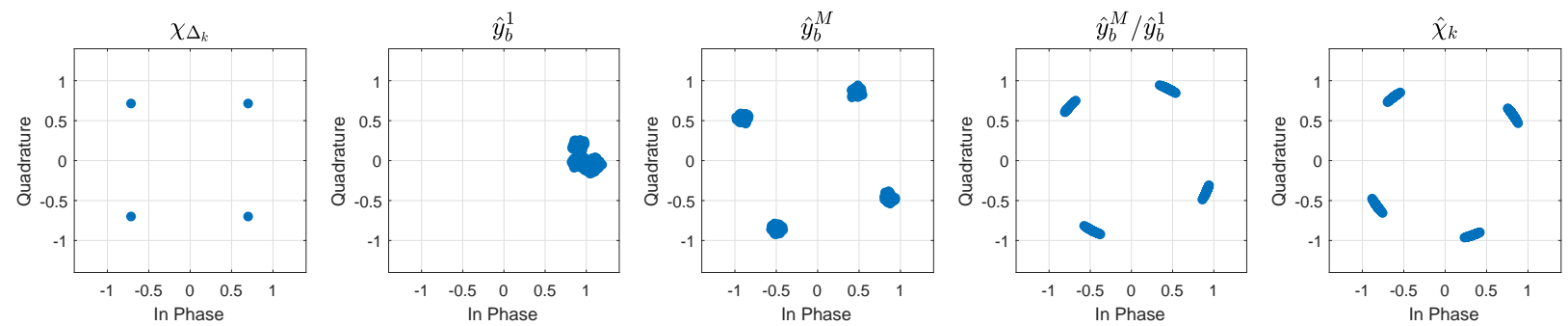

(c)
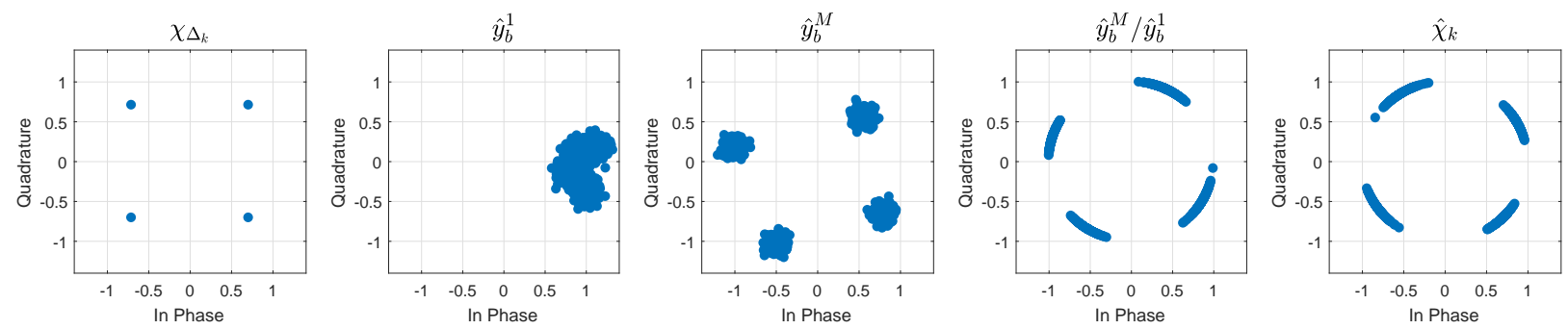

(d)

Fig. 16: Normalized symbol constellation diagrams for Null-Jitter Broadcast technique when code length is (a,b,c) 1024 and (d) 128 under $10 \mathrm{~dB}$ SNR. Communication angle is selected as (a) $60^{\circ}$, (b) $-55^{\circ}$ and (c,d) $-22^{\circ}$. The null jitter angle limit $\theta_{\Delta}$ is chosen as (a,b) $1^{\circ}$ and (c,d) $3.4^{\circ}$.

\section{Conclusions and Future Work}

A JRC system can provide cost-effective platform solution while sensing the environment and allocating the communication links. In this paper, existing coherent MIMO radar waveform generation technique is modified to enable communication. Generated waveforms with this technique do not disturb the orthogonality and transmit beamforming requirements for coherent MIMO radars. Then, using these waveforms three different communication methods are proposed. The specifications for these proposed methods are given in Table.2. Simulations show that the communication performance of the
Code-Driven method outperforms other proposed techniques. Although, Intra-Pulse Communication method provides high data rates, good LPI performance, low receiver complexity, the phase codes of the MIMO radar waveforms are completely dependent on the transmitted information. Thus, pulse to pulse basis coherent processing is limited. On the other hand, other methods MIMO radar waveforms completely independent from information bits and inter-pulse coherent processing can be applicable. Besides, Null-Jitter and Code-Driven methods can be specified as a secure communication. If the receiver does not have the related waveform codes, it can not decipher the information. Although Null-Jitter method delivers information to all 
Table 2: Specifications for Proposed Methods

\begin{tabular}{|c|ccc|}
\hline \multirow{2}{*}{ Parameters } & \multicolumn{3}{|c|}{ Proposed Methods } \\
& Intra-Pulse & Code-Driven & Null-Jitter \\
\hline Radar Waveform \& Information & Dependent & Independent & Independent \\
\hline Communication Type & Directional & Directional & Broadcast \\
\hline Security & None & Secure & Secure \\
\hline Tx/Rx Coherency & None & Required & None \\
\hline LPI Performance & Good & Good & Fair \\
\hline Receiver Complexity & Low & Fair & High \\
\hline Data Rate & $(L-1) \times K \times P R F$ & $K \times P R F$ & $K \times P R F$ \\
\hline Required SNR & Fair & Low & Fair \\
\hline
\end{tabular}

direction except null angle, Code-Driven and Intra-Pulse methods offer directional communication to a specific broadside angle. This makes Code-Driven method more secure.

In order to reach better performance at lower SNR values, instead of using single antenna at the receiver, an antenna array with digital beamforming will improve the performance significantly. For further investigation, mobile communication cases as fading channel effects will be analysed.

\section{References}

[1] B. Paul, A. R. Chiriyath and D. W. Bliss, "Survey of RF Communications and Sensing Convergence Research,' in IEEE Access, vol. 5, pp. 252-270, 2017.

[2] S.D. Blunt, P. Yatham, and J. Stiles, "Intrapulse radar-embedded communications," IEEE Transactions on Aerospace and Electronic Systems, vol. 46, no. 3, pp. 11851200, July 2010.

[3] S. D. Blunt, M. R. Cook, and J. Stiles, "Embedding information into radar emissions via waveform implementation," in Proc. Int. Waveform Diversity $\mathcal{E}$ Design Conf., Niagara Falls, Canada, Aug. 2010, pp. 813.

[4] J. Euziere, R. Guinvarc'h, M. Lesturgie, B. Uguen, R. Gillard, "Dual Function Radar Communication Time-Modulated Array," IEEE Intl. Radar Conf., 2014.

[5] J. Euzire, R. Guinvar'h, I. Hinostroza, B. Uguen and R. Gillard, "Time modulated array for dual function radar and communication," in Proc. of 2015 IEEE International Symposium on Antennas and Propagation $\mathcal{E}$ USNC/URSI National Radio Science Meeting, Vancouver, BC, 2015, pp. 806-807.

[6] A. Hassanien, M. G. Amin, Y. D. Zhang, and F. Ahmad, "A dual function radar-communications system using sidelobe control and waveform diversity," in Proc. IEEE Int. Radar Conf. (RadarCon 2015), Arlington, VA, USA, May 2015,

[7] A. Hassanien, M. G. Amin, Y. D. Zhang, and F. Ahmad, "Dual-function radar-communications: Information embedding using sidelobe control and waveform diversity," IEEE Trans. Signal Processing, vol. 64, no. 8, pp. 21682181 , Apr. 2016.

[8] A. Hassanien, M. G. Amin, Y. D. Zhang, and F. Ahmad, "Signaling strategies for dual-function radar-communications: An overview," IEEE Aerospace and Electronic Systems Magazine, In Press, 2016.

[9] M. Arik and O. B. Akan, "Utilizing sidelobe ASK based joint radarcommunication system under fading," in Proc. of 2017 IEEE Military Communications Conference (MILCOM), Baltimore, MD, 2017, pp. 623628.

[10] A. Hassanien, M. G. Amin, Y. D. Zhang, and F. Ahmad, "A dual-function MIMO radar-communications system using PSK modulation," in Proc. of 2016 24th European Signal Processing Conference (EUSIPCO), Budapest, 2016, pp. 1613-1617.

[11] A. Hassanien, M. G. Amin, Y. D. Zhang, and F. Ahmad, "PhaseModulation Based Dual-Function Radar-Communications," IET Radar, Sonar $\mathcal{E}$ Navigations, 2016.
[12] A. Hassanien, M. G. Amin, Y. D. Zhang, F. Ahmad, and B. Himed, "Non-coherent PSK-based dual-function radar-communication systems," in Proc. IEEE Radar Conf., Philadelphia, PA, May 2016.

[13] Z. Geng, R. Xu, H. Deng, and B. Himed, "Fusion of radar sensing and wireless communications by embedding communication signals into the radar transmit waveform," in IET Radar, Sonar $\mathcal{E}$ Navigation, vol. 12, issue 6, pp. 632-640, June 2018.

[14] P. M. McCormick, S. D. Blunt and J. G. Metcalf, "Simultaneous radar and communications emissions from a common aperture, Part I: Theory," in Proc. of 2017 IEEE Radar Conference (RadarConf), Seattle, WA, 2017, pp. 1685-1690.

[15] P. M. McCormick, B. Ravenscroft, S. D. Blunt, A. J. Duly and J. G. Metcalf, "Simultaneous radar and communications emissions from a common aperture, Part II: Experimentation," in Proc. of 2017 IEEE Radar Conference (RadarConf), Seattle, WA, 2017, pp. 1697-1702.

[16] H. Deng, Z. Geng and B. Himed, "MIMO Radar Waveform Design for Transmit Beamforming and Orthogonality," in IEEE Trans. on Aerospace and Electronic Systems, vol. 52, no. 3, pp. 1421-1433, June 2016.

[17] Z. Geng, H. Deng and B. Himed, "Waveform design for coherent MIMO radar radiation management and transmit-receive beam refocusing," in Proc. of 2016 IEEE Radar Conference (RadarConf), Philadelphia, PA, 2016, pp. $1-5$.

[18] H. Deng and B. Himed, "A Virtual Antenna Beamforming (VAB) Approach for Radar Systems by Using Orthogonal Coding Waveforms," in IEEE Transactions on Antennas and Propagation, vol. 57, no. 2, pp. 425435, Feb. 2009.

[19] M. J. Nowak, Zhiping Zhang, Yang Qu, D. A. Dessources, M. Wicks and Zhiqiang Wu, "Co-designed radar-communication using linear frequency modulation waveform," in Proc. of MILCOM 2016 - 2016 IEEE Military Communications Conference, Baltimore, MD, 2016, pp. 918-923. 